\title{
Roof Planes Detection Via a Second-order Variational Model
}

\author{
Battista Benciolini ${ }^{\mathrm{a}}$, Valeria Ruggiero ${ }^{\mathrm{c}},{\text { Alfonso } \text { Vitti }^{\mathrm{a}} \text {, Massimo Zanetti }}^{\mathrm{b}}$ \\ ${ }^{a}$ University of Trento - DICAM, I-38123 Trento, Italy \\ ${ }^{b}$ University of Trento - DISI, I-38123 Trento, Italy \\ ${ }^{c}$ University of Ferrara - DMI, I-44122 Ferrara, Italy
}

\begin{abstract}
The paper describes a unified automatic procedure for the detection of roof planes in gridded height data. The procedure exploits the Blake-Zisserman (BZ) model for segmentation in both $2 \mathrm{D}$ and $1 \mathrm{D}$, and aims to detect, to model and to label roof planes.

The BZ model relies on the minimization of a functional that depends on first- and second-order derivatives, free discontinuities and free gradient discontinuities. During the minimization, the relative strength of each competitor is controlled by a set of weight parameters. By finding the minimum of the approximated BZ functional, one obtains: 1) an approximation of the data that is smoothed solely within regions of homogeneous gradient, and 2) an explicit detection of the discontinuities and gradient discontinuities of the approximation.

Firstly, input data is segmented using the 2D BZ. The maps of data and gradient discontinuities are used to isolate building candidates and planar patches (i.e. regions with homogeneous gradient) that correspond to roof planes. Connected regions that can not be considered as buildings are filtered according to both patch dimension and distribution of the directions of the normals to the boundary. The $1 \mathrm{D}$ BZ model is applied to the curvilinear coordinates of boundary points of building candidates in order to reduce the effect of data granularity when the normals are evaluated. In particular, corners are preserved and can be detected by means of gradient discontinuity.

Lastly, a total least squares model is applied to estimate the parameters of the plane that best fits the points of each planar patch (orthogonal regression with planar model). Refinement of planar patches is performed by assigning those points that are close to the boundaries to the planar patch for which a
\end{abstract}


given proximity measure assumes the smallest value. The proximity measure is defined to account for the variance of a fitting plane and a weighted distance of a point from the plane.

The effectiveness of the proposed procedure is demonstrated by means of its application to urban digital surface models characterized by different spatial resolutions. Results are presented and discussed along with some promising developments.

Keywords: Segmentation, Variational model, DSM processing, Edge and crease detection, Filtering, Roof planes, Plane detection and modeling

\section{Introduction}

\subsection{The detection roof planes}

The identification of roof planes from the analysis of three dimensional (3D) point clouds and Digital Surface Models (DSMs) is an active research

and application topic (Rottensteiner et al., 2014). The representation of building volumes in 3D city models can be improved when roof planes are properly included in the models. City models and DSMs of urban areas are widely used in several applications, e.g. acoustic and energy studies (Jochem et al., 2009), environmental and pollution modeling, visualization, cadastre updating and building volume estimates, urban and land planning, cultural heritages studies, telecommunication networks design and orthophoto generation in urban contexts (Habib and Kim, 2006; Barazzetti et al., 2010).

From a general point of view, the processing of elevation data of urban areas based on the so-called data-driven approach involves data segmentation, enforcement of topological consistency if needed, object detection and reconstruction. Many different strategies have been proposed to tackle the mentioned tasks (Haala and Kada, 2010). The model-driven approach is an alternative to the use of data-driven methods (Tarsha-Kurdi et al., 2007b). For example, Huang et al. (2013) used a generative model based on a primitive library for roof detection and reconstruction. Works mixing the bottomup and the top-down approaches have also been presented (e.g. Satari et al., 2012) along with strategies involving the integrated processing of different types of data, such as topographic maps or imagery data (Brenner, 2005; Rottensteiner, 2010; Demir and Baltsavias, 2012; Awrangjeb et al., 2013).

In this work, a procedure for the detection of roof planes in gridded data is presented. As suggested by Vitti (2012b), regions with homogeneous 
gradient (i.e. planar patches) and their boundaries (i.e. roof edges and ridges) can be detected by exploiting the global variational model of the second order proposed by Blake and Zisserman (1987). The application of the model to DSMs was investigated in two preliminary works by Zanetti and Vitti (2013) and Benciolini and Vitti (2015); recently Zanetti et al. (2016) presented numerical results useful for practical purposes. This work presents a complete procedure for accomplishing the specific goal of detecting and labeling roof planes.

The paper is organized as follows. In the remainder of this section references to know solutions which have some kind of relationship with the propose procedure are provided and the main features justifying the procedure based on variational segmentation are introduced. The BZ model and its numerical implementation are presented in Section 2. A detailed step-bystep description of the automatic roof-detection procedure is given in Section 3. Other models involved in the procedure are also presented therein. To illustrate the procedure outputs a sample application to a DSM (1 m spatial resolution) of an urban area of the city of Trento, Italy, is considered. Section 4 presents a detailed application of the procedure to a DSM $(25 \mathrm{~cm}$ spatial resolution) of an urban area of the city of Vaihingen, Germany. Final remarks and perspectives on further developments are given in the Section 5 .

\subsection{Known solutions}

The segmentation of 3D point clouds and DSMs can be addressed applying different data-driven methods directly to height values, normals or other features derived from height values. Strategies based on clustering, region growing, edge and contour based methods have been proposed along with others based on parametric or statistical models. In this context, interesting examples are proposed in the following works. In (Ohtake et al., 2004) edge detection on triangle meshes is performed by analyzing the principal curvature and its derivatives. In (Rottensteiner, 2003) a region growing model is applied to normal vectors for the generation of 3D building models. In (Wang et al., 2013) normal vectors are treated as points on the unit sphere and then clustered to detect planes and other regular surfaces. Filin and Pfeifer (2006) proposed a feature-based approach to cluster Airborne Laser Scanning (ALS) data, capable of detecting planar elements; in that work, normal vectors were computed according to a slope adaptive neighborhood mechanism. Biosca and Lerma (2008) used the fuzzy clustering method and 
the so called possibilistic $c$-means mode-seeking algorithm to detect planes in point clouds. Sampath and Shan (2010) proposed a procedure for the segmentation and reconstruction of polyhedral roofs based on the eigen-analysis to filter planar and non-planar (set of) points and on a fuzzy $k$-means method to cluster planar points.

Other works present conceptual or operational resemblances. For example in (Lavoué and Dupont, 2009) a polygonal mesh is approximated by a set of semi-sharp surfaces built after the detection of the main linear features of the analyzed object; in the same work a global model based on the Variational Shape Approximation by Cohen-Steiner et al. (2004) is used to produce a piecewise smooth surface segmentation with sharp edges. In (Goebbels and Pohle-Fröhlich, 2016) the reconstruction of complex roofs is achieved starting from an ad-hoc interpolated height map and using image processing techniques to avoid shortcomings of standard geometric methods for plane detection. Lin et al. (2014) applied the principal component analysis to a weighted covariance matrix with a geometric median to compute local geometric characteristics less sensitive to data noise and partial sampling. Jochem et al. (2012) applied a 3D $k$-nearest neighbors based region growing model to normals for the segmentation of planes in large ALS data set. Chen et al. (2012) applied a region growing algorithm based on a plane-fitting technique to select building points in ALS data followed by an adaptive implementation of the RANSAC algorithm (Fischler and Bolles, 1981) to segment building roof-tops. Plane detection can also be performed by means of the Hough transform (Hough, 1962), (e.g. Tarsha-Kurdi et al., 2007a; Borrmann et al., 2011). Kim and Shan (2011) based the modeling of building roofs on the application of the level set method to the normals for the segmentation of planes in ALS data. Recently, Yan et al. (2014) adopted a global plane-fitting approach based on the works by Delong et al. (2012) and Hossam and Boykov (2012) for roof segmentation in LiDAR point clouds.

\subsection{A novel approach based on 2nd order variational segmentation}

The rationale of the proposed approach can be understood by considering that roof planes are basically planar surfaces and that buildings have regular shapes, e.g. polygonal footprints. In fact, a complex roof can be thought of as a piecewise smooth surface with edges and creases, i.e. surface discontinuities and surface gradient discontinuities respectively (see Figure 1(a) and $1(b))$. The projection of the roof boundary on the ground is the building footprint and it can be identified as an area surrounded by a closed curve 
made up of edge points (see Figure 1(c)). Within a building footprint, edge and crease (edge\&crease) discontinuities allow for the representation of the closed boundary of each planar patch that comprises the complex roof (see Figure 1(d)). In real cases, noisy data and discrete sampling affect the quality of LiDAR point clouds and gridded DSMs. The noise affecting data should be reduced without altering edge and crease structures that would help the detection of planar patches. Due to the spatial granularity of the data, the detection of regular shapes is difficult and modeling a boundary with a smooth approximation with sharp corners would be very useful in this case. An example of a convenient piecewise linear approximation of the gridded points representing a building boundary is given in Figure 2.

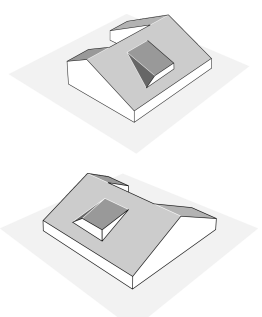

(a)

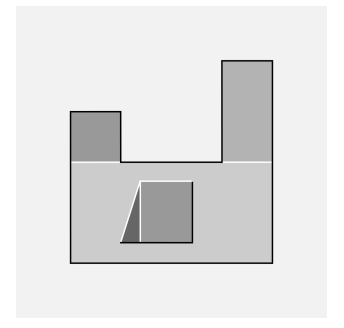

(b)

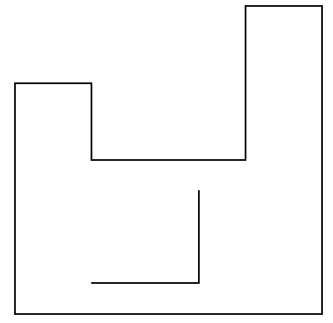

(c)

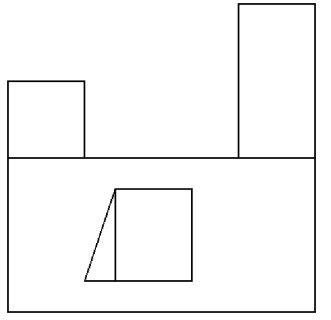

(d)

Figure 1: A complex roof with one garret: (a) 3D view; (b) top view; (c) edge points of the roof surface; (d) edge\&crease points of the roof surface.

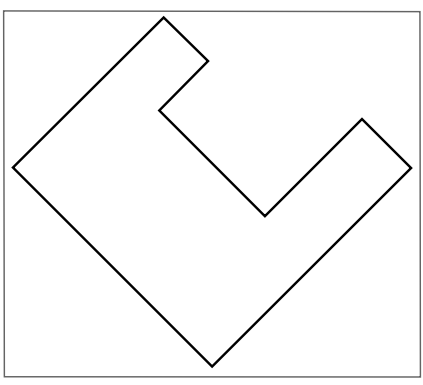

(a)

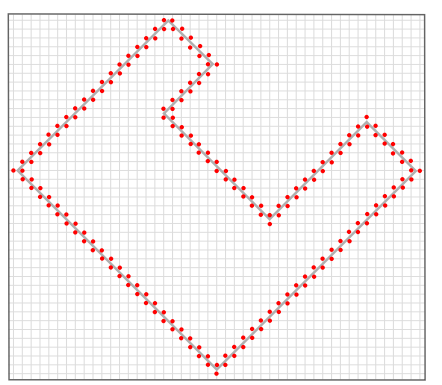

(b)

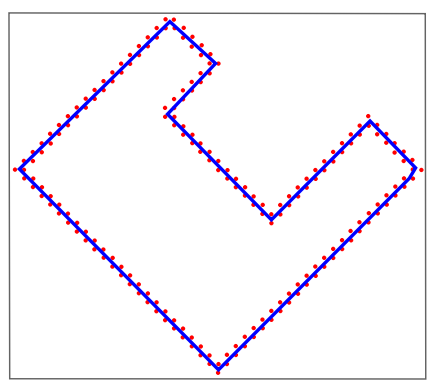

(c)

Figure 2: Building footprint approximation: (a) real footprint; (b) gridded sampling; (c) piecewise smooth recovery of the boundary.

The proposed approach to building and roof plane detection exploits an elliptic approximation of a variational model by Blake and Zisserman (1987). 
The model is applied in 2D to approximate a DSM by piecewise nearlylinear surfaces and in 1D to approximate footprint boundaries by piecewise nearly-linear curves.

The 2D BZ applied to an input DSM returns a piecewise nearly-linear approximation of data and the graphs of two auxiliary functions mapping the discontinuity of the smooth approximation and of its gradient (edges and creases). Such outputs provide the ideal starting point for a further process that implements the concepts given above. The discontinuity map is first processed to isolate building footprint candidates. The candidates are then filtered according to their area and the direction of the normals to the boundary. The DSM sampling-interpolation noise can significantly affect the distribution of the normals, therefore the 1D BZ is applied to each building footprint candidate in order to model its boundary by means of a piecewise nearly-linear approximation with sharp corners (see Benciolini and Vitti, 2015). The rejection of a candidate is performed on the assumption that buildings have, in general, polygonal footprints with right angles. Both the use of discrete differential operators and the smoothing of building boundaries produce ambiguity in assigning pixels on the borders to the adjacent regions (planar patches). Such ambiguity is resolved by exploiting height values of the piecewise nearly-linear approximation and the Total Least Squares (TLS) model for fitting roof plane points. According to the values of a specific proximity measure that depends on the variance of the fitting plane and the (weighted) orthogonal distance of a point from a plane, planar patches are refined to properly include boundary points.

As described, roof detection would strongly benefit from a proper analysis of data and gradient discontinuities of a smooth approximation of the input DSM. However, basic thresholding methods on the discrete gradient and Hessian images for identifying discontinuities are very unstable and sensitive to noise Yazid and Arof (2013); Harris and Stephens (1988); Lindeberg (1998). The application of these methods always requires a-priori information and their outcome is not sufficiently complete to perform the analysis proposed in this work.

The overall procedure based on the outcome of the BZ segmentations presents several nice features which can be appreciated also by referring to properties of different known approaches.

With reference to procedures based on the analysis of elevation data, such as region growing, RANSAC and eigen-analysis methods, in the propose 
procedure specific selections of neighborhood points are not necessary; $a$ priori information about the total number of planes to be detected or seed initialization are also non necessary.

The proposed approach implicitly avoids the smoothing of sharp features, it is rather robust to outliers and less sensitive to noise with respect to methods relying on principal component analysis.

With respect to procedures based on the analysis in feature domains, such as those based on clustering methods and the Hough transform, in the proposed procedure spatially separated coplanar and parallel patches are detected without the need of specific modeling or prior assumptions. Moreover, all the planar patches are detected in a single step.

Topological consistency of roof planes is obtained without the need of specific processing. Complex roofs can be detected by exploiting second order information and a detailed descriptions of their structure can be obtained exploiting the discontinuity maps (see for instance the garret in Figure 1) instead of limited libraries of geometric primitives or object definitions.

Parental relationship of roof planes and building footprints is obtained very naturally by the analysis of the discontinuity maps without the need of $a$ priori information, such as building footprints. A Digital Terrain Model (DTM) is not necessary to separate ground and non-ground regions.

As shown in (Zanetti and Vitti, 2013), the BZ model can be used alone as an up to the second-order edge-preserving noise-removal model. The output of the BZ model could however be used also in analyses where pre-processing is required to help the top-down reconstruction modeling, e.g. in (Huang et al., 2013).

\section{The Blake-Zisserman 2nd-order variational model}

Essential information about the BZ model and its numerical implementation are provided in this section. The BZ model comes from the literature of Visual Reconstruction and it allows for the approximation of an input signal by a piecewise smooth function with discontinuities. Although the variational model can be formulated in any dimension $n$, it finds its natural application with $n=1,2$, i.e. when the input data is either a signal or an image, respectively. 
To better explain the features of the BZ model, an overview of the firstorder variational model by Mumford-Shah is given along with basic concepts of variational segmentation. Then, the BZ model, which overcomes many limitations imposed by the MS and generates a richer outcome, is described.

Mumford and Shah (1989) proposed a model for image segmentation based on an energy functional depending on first-order derivatives and free discontinuities. Let us consider a gray-scale image as a function $g$ defined on a two dimensional open set $\Omega \subset \mathbb{R}^{2}$ (usually a rectangle). The aim is to find $u$ (a regularized version of $g$ ) and $K$ (a compact one-dimensional set $K \subset \bar{\Omega}$ ) such that $u$ is close to $g$ and as smooth as possible on $\Omega \backslash K$, therefore allowing discontinuities of $u$ on $K$. Heuristically, this can be achieved by minimizing the so-called Mumford-Shah (MS) functional

$$
\mathcal{M S}(u, K):=\mu \int_{\Omega}|u-g|^{2} d x+\int_{\Omega \backslash K}|\nabla u|^{2} d x+\alpha \mathcal{H}^{1}(K \cap \Omega)
$$

among all the functions differentiable outside $K$, i.e. $u \in C^{1}(\Omega \backslash K)$, where $K \subset \bar{\Omega}$ is compact. Here, $\mathcal{H}^{1}$ is the 1 -dimensional Hausdorff measure, and $\alpha, \mu$ are positive parameters. During the minimization, the three terms provide different contributions to the properties of the final minimizer: the first term keeps $u$ close to the original datum $g$; the second term forces $u$ to be smooth outside $K$; the third term forces $K$ to be a one-dimensional set with finite length. The weight of each term in the minimization is controlled by the parameters $\alpha, \mu$. The Mumford-Shah model involves both surface and line energies whose support depends on an unknown discontinuity set. This represents a typical example of a general class of problems that De Giorgi (1991) characterized as free discontinuity problems. These models are widely employed for signal approximation (one dimension), image segmentation (two dimensions), and fracture analysis (three dimensions). Other variational models of the first-order, such as Total Variation (Rudin et al., 1992; Chan et al., 2006) and Anisotropic Diffusion (Perona and Malik, 1990), exist and are widely used, along with the MS model, to segment various types of data in different application fields.

Despite the given model being very convincing, the existence of a minimizer that makes sense for real applications is not yet ensured. Nonetheless, classical methods of Calculus of Variations can not be applied to prove the existence of a minimum. To overcome this problem De Giorgi et al. (1989) proposed the use a functional dependent sole on $u$ and to recover the set of contours $K$ via the discontinuity set of $u$. De Giorgi proposed a weak formu- 
lation of the problem in the space of Special Functions of Bounded Variation $S B V(\Omega)$ :

$$
\mathcal{E}(u):=\int_{\Omega}\left\{\mu|u-g|^{2}+|\nabla u|^{2}\right\} d x+\alpha \mathcal{H}^{1}\left(S_{u} \cap \Omega\right),
$$

where $u \in S B V(\Omega)$, and $S_{u}$ is discontinuity set of $u$. Using compactness in $S B V(\Omega)$ and lower semi-continuity theorems, Ambrosio et al. (2000) showed that under mild conditions, there exists a solution such that $\mathcal{H}^{1}\left(S_{u}\right)<\infty$. Moreover, by regularity results one has that the couple $\left(u, \overline{S_{u}}\right)$ can be identified with a minimizer of the strong formulation De Giorgi et al. (1989).

The numerical computation of a function $u$ that achieves a minimum of the energy (2) can not be addressed by differentiation because of the onedimensional measure term $\mathcal{H}^{1}\left(S_{u} \cap \Omega\right)$. Ambrosio and Tortorelli (1992), by taking advantage of a result by Modica and Mortola (1977), proposed a way to approximate the discontinuity set by adding an auxiliary function $s$ (that plays the role of indicator function) to the model. The convergence of their approximated (relaxed) model to (2) is formalized via $\Gamma$-convergence, which is a notion of convergence suitable for functionals (De Giorgi and Franzoni, 1975; Braides, 2002). A description of the long procedure that leads to the practical use of the MS model can be found in (Vitti, 2012b). Details concerning a similar procedure necessary for the application of the BZ model follows in this Section.

The MS model suffers some side effects precisely because of its first-order nature. Because of the one-dimensional geometric term, the discontinuity set of the solution is the union of $C^{1}$ arcs. The minimization of the length of the discontinuity set forces triple-junctions to meet always at $2 / 3 \pi$ wide angles, thus the real geometry of boundaries may be lost. Moreover, because of the minimization of the gradient norm, the solution can only be a piecewise near-constant approximation of the data. Actually, in regions where the data presents a steep gradient the functional minimization gives rise to a sort of staircase-like approximation. This phenomenon is well-known as over-segmentation of steep gradients, and if triggered, it causes the failure of segmentation as it introduces many fictitious discontinuities. Since only the gradient norm of the MS approximation is controlled, features such as discontinuities of the first derivative of the approximation can not be detected. Clearly, this kind of features are of great interest in the context of analysis of urban hight data. These facts led to the consideration of a more general variational model which was introduced by Blake and Zisserman (1987) 
to overcome the limitations of the MS model. In the BZ formulation, the squared norm of the Hessian matrix is considered instead of the gradient component. The minimization of this 2 nd order term allows to find a solution that is basically a piecewise nearly-linear approximation of the data.

In this context, the image denoising model based on the concept of total generalized variation (TGV) is a different variational formalization of a high order segmentation model that leads to a piecewise smooth restoration not affected by the spurious staircasing effect (Bredies et al., 2010). By means of automatic balancing of the first to the $k^{\text {th }}$ derivatives of the recovering function $u$, the TGV model can be seen as a generalization of the well-know total variation (TV) approach based on the bounded variation seminorm (Rudin et al., 1992). With respect to TGV, in the BZ model the discontinuity sets of the recovering function $u$ are explicitly handled and are, in fact, a distinctive feature of the BZ solution. In this work the set of the discontinuities of $s, z$ are indeed widely exploited along with the regularized solution $u$. A similar argument holds for the TV and the MS models; in the MS model the presence of the geometric term permits to produce segmentations that differ from those produced by the TV model. The possibility to control the measure of the discontinuity set, for example, can permit to control the number of spurious jumps introduced because of the staircasing effect.

The Blake-Zisserman functional formulation is given as minimization of

$$
\begin{aligned}
\mathcal{B Z}\left(u, K_{0}, K_{1}\right) & :=\mu \int_{\Omega}|u-g|^{2} d x+\int_{\Omega \backslash\left(K_{0} \cup K_{1}\right)}\left|\nabla^{2} u\right|^{2} d x+ \\
& +\alpha \mathcal{H}^{1}\left(K_{0} \cap \Omega\right)+\beta \mathcal{H}^{1}\left(\left(K_{1} \backslash K_{0}\right) \cap \Omega\right),
\end{aligned}
$$

among all $u \in C^{2}\left(\Omega \backslash\left(K_{0} \cup K_{1}\right)\right) \cap C^{0}\left(\Omega \backslash K_{0}\right)$ and compact sets $K_{0}, K_{1}$ such that $K_{0} \cup K_{1}$ is closed in $\bar{\Omega} . \quad \mu, \alpha, \beta$ are positive parameters. The solution is required to be twice differentiable outside $K_{0} \cup K_{1}$ and at least differentiable outside $K_{0}$. Therefore, discontinuities are allowed both on $K_{0}$ and $K_{1}$, whereas discontinuities of the gradient are allowed only on $K_{1}$. The set $K_{1} \backslash K_{0}$ is the set of crease points of $u ; \alpha$ and $\beta$ are contrast parameters regulating the total length of the discontinuity sets.

Existence theory for this formulation have been stated by Carriero et al. (1997). The authors have considered the weaker problem of minimizing the functional

$$
\mathcal{F}(u):=\int_{\Omega}\left\{\mu|u-g|^{2}+\left|\nabla^{2} u\right|^{2}\right\} d x+\alpha \mathcal{H}^{1}\left(S_{u}\right)+\beta \mathcal{H}^{1}\left(S_{\nabla u} \backslash S_{u}\right),
$$


among any $u \in G S B V^{2}(\Omega)$, a proper subspace of the class of Generalized Special Functions of Bounded Variation. The choice of $G S B V^{2}(\Omega)$ is necessary for having a proper definition of $\nabla^{2} u$ and $S_{\nabla u}$, the discontinuity set of $\nabla u$. A minimizer of (4) exists and it can be identified with a minimizing couple of the strong formulation provided $\beta \leq \alpha \leq 2 \beta$. It is worth noting that now in (4) there is only the unknown $u$.

Similarly to the MS case, a direct differentiation of $\mathcal{F}$ is not possible because of the one-dimensional measure terms. Ambrosio et al. (2001), by properly adapting the work by Ambrosio and Tortorelli (1992), introduced two auxiliary functions $s, z: \Omega \rightarrow[0,1]$ to the model and proposed a $\Gamma$ convergence approximation of $\mathcal{F}$ via the family of elliptic functionals

$$
\begin{aligned}
\mathcal{F}_{\epsilon}(u, s, z) & :=\mu \int_{\Omega}|u-g|^{2} d x+\int_{\Omega} z^{2}\left|\nabla^{2} u\right|^{2} d x+ \\
& +\xi_{\epsilon} \int_{\Omega}\left(s^{2}+o_{\epsilon}\right)|\nabla u|^{2} d x+ \\
& +(\alpha-\beta) \int_{\Omega}\left[\epsilon|\nabla s|^{2}+\frac{1}{4 \epsilon}(s-1)^{2}\right] d x+ \\
& +\beta \int_{\Omega}\left[\epsilon|\nabla z|^{2}+\frac{1}{4 \epsilon}(z-1)^{2}\right] d x,
\end{aligned}
$$

defined on proper Sobolev spaces. Here, $\epsilon$ is the $\Gamma$-convergence parameter and the convergence is intended for $\epsilon \rightarrow 0 ; \xi_{\epsilon}, o_{\epsilon}$ are infinitesimals of higher order than $\epsilon$. The parameters $\mu, \alpha, \beta$ are all positive and they control the relative strength of the functional components. The functional (5) approximates (4) in the sense of $\Gamma$-convergence: for any $\epsilon>0$ the functional $\mathcal{F}_{\epsilon}$ always admits a minimizing triplet, namely $\left(u_{\epsilon}, s_{\epsilon}, z_{\epsilon}\right)$, and by sending $\epsilon \rightarrow 0$, the $\Gamma$ convergence ensures that the sequence $\left\{\left(u_{\epsilon}, s_{\epsilon}, z_{\epsilon}\right)\right\}_{\epsilon>0}$ converges to a triplet $\left(u^{*}, s^{*}, z^{*}\right)$, where $u^{*}$ is a minimizer of the limit functional $\mathcal{F}$ and $s^{*}, z^{*} \equiv 1$ almost everywhere on $\Omega$. In the variational approximation $\mathcal{F}_{\epsilon}$ only integral terms defined over the entire domain $\Omega$ are involved. The last two new integrals in (5) that involve the functions $s$ and $z$ are convex and, as $\epsilon \rightarrow 0$, proved to $\Gamma$-converge to the last two terms of $\mathcal{F}$, where such latter terms measure the size of the discontinuity sets of $u$ and $\nabla u$ respectively. The approximation of the length of a curve by means of a surface integral involving an auxiliary function defined over a domain $\Omega$ was proposed by Modica and Mortola (1977) who proved a conjecture proposed by De Giorgi and Franzoni (1975) (see Modica and Mortola, 1977, and references therein). The advantage of formulation (5) is that the functional can be numerically addressed as detailed in Section 2.2. 


\subsection{Geometrical behavior of a minimizer}

By analyzing the role of each term in the minimization of (5) it is possible to predict, at least in a qualitative way, the geometrical behavior of a minimizing triplet $\left(u_{\epsilon}, s_{\epsilon}, z_{\epsilon}\right)$, when $\epsilon$ approaches 0 . This understanding is fundamental in order to properly exploit the potential of the BZ model for solving the roof-detection problem. Indeed, the outcome of the minimization provides all the elements needed to apply the reasoning given in Section 1.3.

Because of the minimization, the closeness term $\left|u_{\epsilon}-g\right|^{2}$ forces the function $u_{\epsilon}$ to fit the original datum $g$. The smoothness constraint $\left|\nabla^{2} u_{\epsilon}\right|^{2}$ guarantees the approximating function $u_{\epsilon}$ to be piecewise nearly-linear (null Hessian), see Figure 3 (upper graph). Discontinuities of first and second order are allowed only along 1-dimensional sets with finite length and they are properly detected by means of the functions $s_{\epsilon}$ and $z_{\epsilon}$, respectively:

- In order to keep the term $\left(s_{\epsilon}-1\right)^{2} / 4 \epsilon$ bounded, $s_{\epsilon}$ must be 1 almost everywhere over $\Omega$. This condition is violated only at those points where it is energetically more convenient to suppress the $s_{\epsilon}^{2}\left|\nabla u_{\epsilon}\right|^{2}$ component (the only other competitor containing $s_{\epsilon}$ ), by letting $s_{\epsilon}=0$. It is important to note that the difference $\alpha-\beta$ controls the ratio at which this mechanism takes place. Keeping the term $\epsilon\left|\nabla s_{\epsilon}\right|^{2}$ bounded avoids large oscillations of the function $s_{\epsilon}$, hence the transition of $s_{\epsilon}$ between 0 and 1 turns out to be smooth, see Figure 3 (central graph). As a result, $s_{\epsilon}$ is approximately 0 in a tubular neighborhood of the 1-variety $S_{u}$ (the discontinuity set of $u$ ). The radius of the tubular neighborhood shrinks as $\epsilon \rightarrow 0$.

- A similar argument can be stated for $z_{\epsilon}$. Because of the competition between the terms $\left(z_{\epsilon}-1\right)^{2} / 4 \epsilon$ and $z_{\epsilon}^{2}\left|\nabla^{2} u_{\epsilon}\right|^{2}$, it is expected that $z_{\epsilon}$ is approximately 0 in a tubular neighborhood of the 1-variety $S_{\nabla u} \cup S_{u}$, the joint discontinuity set of $u$ and $\nabla u$, see Figure 3 (bottom graph).

From the discussion above it follows that, for small values of $\epsilon$, a minimizer $u_{\epsilon}$ of $\mathcal{F}_{\epsilon}$ is an approximation (in the $\Gamma$-convergence sense) of a minimizer $u$ of $\mathcal{F}$, whereas $s_{\epsilon}, z_{\epsilon}$ are smooth approximations of the indicator functions of the discontinuity sets $S_{u}$ and $S_{\nabla u} \cup S_{u}$, respectively. The points where the two auxiliary functions are nearly 0 represent the (first and second order) discontinuity points of $u$. 


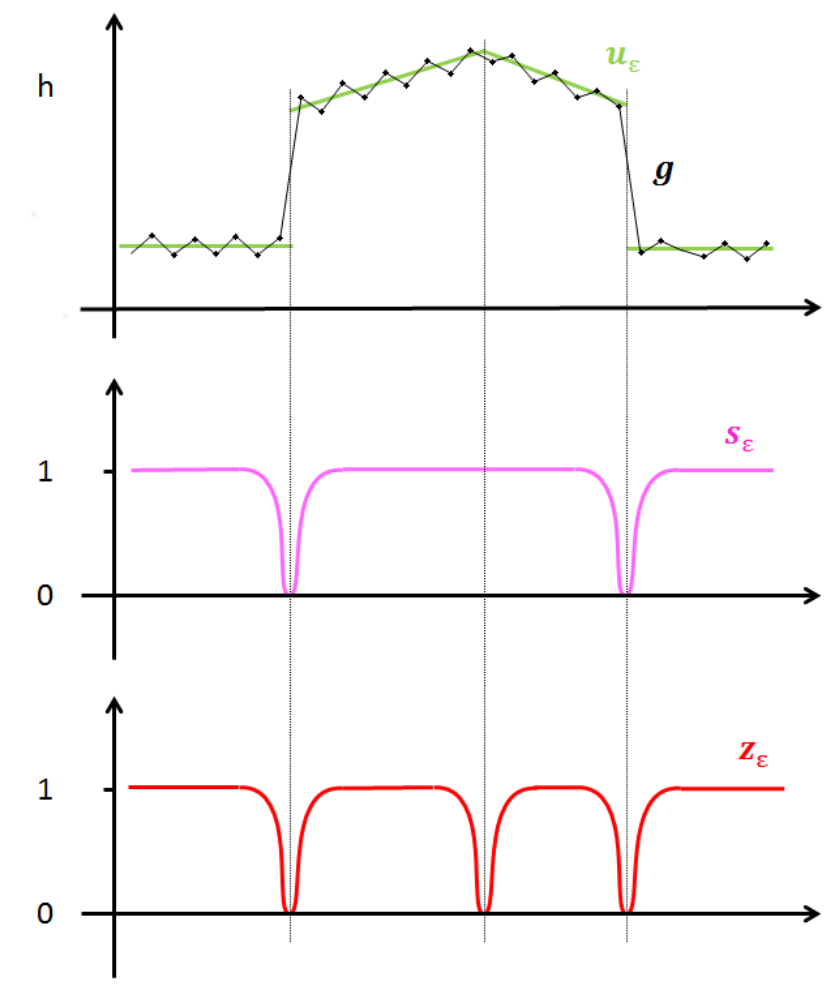

Figure 3: Geometrical behavior of a minimizer on a typical urban shape. A slice of the minimizing functions $u_{\epsilon}, s_{\epsilon}, z_{\epsilon}$ is represented. In the upper graph the (noisy) input data $g$ and its smooth approximation $u_{\epsilon}$. In the middle graph the edge-detector function $s_{\epsilon}$, and in the lower graph the edge\&crease detector function $z_{\epsilon}$.

\subsection{Numerical implementation of the 2D Blake-Zisserman model}

In view of the discussion given above, a strategy for numerically computing a minimizer of the functional $\mathcal{F}_{\epsilon}$, for fixed $\epsilon>0$ is needed. In this work the strategy proposed by Zanetti et al. (2016) is adopted given its efficiency. In the following an overview of the strategy is given.

The cross terms $z^{2}\left|\nabla^{2} u\right|^{2}$ and $\left(s^{2}+o_{\epsilon}\right)|\nabla u|^{2}$ do not allow for the definition of a single gradient descent direction. However, a partial descent minimization is possible because of nice properties of the functional. Let us discretize the rectangular data domain $\Omega \subset \mathbb{R}^{2}$ by a lattice of points $\Lambda=\left\{\left(i t_{x}, j t_{y}\right): \forall i=1, \ldots, N, \forall j=1, \ldots, M\right\}$, where $t_{x}$ and $t_{y}$ are the step sizes on the $x$ and $y$ directions. The known values of the data $g$ on the grid points $(i, j)$ are denoted $g_{i j}$. Similarly, the values of the functions 
$u, s, z$ on the points of the grid are denoted $u_{i j}, s_{i j}, z_{i j}$. For any function $v \in\{g, u, s, z\}$, let $\mathbf{v}$ be the column vector of size $N \cdot M$ obtained from $v_{i j}$ by a column-wise vectorization. Given any pair of indices $(i, j)$, the function $w(i, j):=(j-1) N+i$ returns the position index of the quantity $v_{i j}$ in the vector $\mathbf{v}$. Shortly, $[\mathbf{v}]_{w(i, j)}=v_{i j}$. The differential operators $\nabla v, \nabla^{2} v$ can be implemented in a discrete way using difference-schemes. Using the tensor product the matrix operators $\mathbf{D}_{x}, \mathbf{D}_{y}, \mathbf{D}_{x x}, \mathbf{D}_{y y}, \mathbf{D}_{x y}$ are defined in such a way that:

$$
\begin{aligned}
& \frac{v_{i+1, j}-v_{i, j}}{t_{x}}=\left[\mathbf{D}_{x} \mathbf{v}\right]_{w(i, j)} \\
& \frac{v_{i, j+1}-v_{i, j}}{t_{y}}=\left[\mathbf{D}_{y} \mathbf{v}\right]_{w(i, j)} \\
& \frac{v_{i+1, j}-2 v_{i, j}+v_{i-1, j}}{t_{x}^{2}}=\left[\mathbf{D}_{x x} \mathbf{v}\right]_{w(i, j)} \\
& \frac{v_{i, j+1}-2 v_{i, j}+v_{i, j-1}}{t_{y}^{2}}=\left[\mathbf{D}_{y y} \mathbf{v}\right]_{w(i, j)} \\
& \frac{v_{i+1, j+1}-v_{i, j+1}}{t_{x} t_{y}}-\frac{v_{i+1, j}-v_{i, j}}{t_{x} t_{y}}=\left[\mathbf{D}_{x y} \mathbf{v}\right]_{w(i, j)}
\end{aligned}
$$

with homogeneous Neumann boundary conditions.

Let $\mathbf{v}^{2}$ be the vector whose entries are the squared elements of the vector $\mathbf{v}$. The Euclidean norm of a vector $\mathbf{x}=\mathbf{A v}$ can be computed as $|\mathbf{x}|^{2}=\mathbf{x}^{T} \mathbf{x}=$ $(\mathbf{A v})^{T}(\mathbf{A v})=\left(\mathbf{v}^{T} \mathbf{A}^{T}\right)(\mathbf{A v})$. Let $\mathbf{M}_{\mathbf{v}}$ denote the diagonal matrix whose diagonal entries are the elements of the vector $\mathbf{v}$ and the off-diagonal entries are zero. Let us denote $\mathbf{e}=(1,1, \ldots, 1)^{T}$ the vector of size $N \cdot M$ with all entries equal to 1 . The discretization of the functional (5) can be written as:

$$
\begin{aligned}
F_{\epsilon}(\mathbf{u}, \mathbf{s}, \mathbf{z})= & \mu(\mathbf{u}-\mathbf{g})^{T}(\mathbf{u}-\mathbf{g})+ \\
& +\left\{\left(\mathbf{D}_{x x} \mathbf{u}\right)^{T} \mathbf{M}_{\mathbf{z}^{2}}\left(\mathbf{D}_{x x} \mathbf{u}\right)+\left(\mathbf{D}_{y y} \mathbf{u}\right)^{T} \mathbf{M}_{\mathbf{z}^{2}}\left(\mathbf{D}_{y y} \mathbf{u}\right)+\right. \\
& \left.+2\left(\mathbf{D}_{x y} \mathbf{u}\right)^{T} \mathbf{M}_{\mathbf{z}^{2}}\left(\mathbf{D}_{x y} \mathbf{u}\right)\right\}+ \\
& +\xi_{\epsilon}\left\{\left(\mathbf{D}_{x} \mathbf{u}\right)^{T} \mathbf{M}_{\mathbf{s}^{2}}\left(\mathbf{D}_{x} \mathbf{u}\right)+\left(\mathbf{D}_{y} \mathbf{u}\right)^{T} \mathbf{M}_{\mathbf{s}^{2}}\left(\mathbf{D}_{y} \mathbf{u}\right)\right\}+ \\
& +(\alpha-\beta)\left\{\epsilon\left[\left(\mathbf{D}_{x} \mathbf{s}\right)^{T}\left(\mathbf{D}_{x} \mathbf{s}\right)+\left(\mathbf{D}_{y} \mathbf{s}\right)^{T}\left(\mathbf{D}_{y} \mathbf{s}\right)\right]+\frac{1}{4 \epsilon}(\mathbf{s}-\mathbf{e})^{T}(\mathbf{s}-\mathbf{e})\right\}+ \\
& +\beta\left\{\epsilon\left[\left(\mathbf{D}_{x} \mathbf{z}\right)^{T}\left(\mathbf{D}_{x} \mathbf{z}\right)+\left(\mathbf{D}_{y} \mathbf{z}\right)^{T}\left(\mathbf{D}_{y} \mathbf{z}\right)\right]+\frac{1}{4 \epsilon}(\mathbf{z}-\mathbf{e})^{T}(\mathbf{z}-\mathbf{e})\right\} .
\end{aligned}
$$


The functional $F_{\epsilon}$ is quadratic in any of the variables $\mathbf{u}, \mathbf{s}, \mathbf{z}$, when the others are kept fixed. Therefore, gradient equations with respect to these three variables are linear equations and they can be written as:

$$
\begin{aligned}
& \nabla_{u} F_{\epsilon}(\mathbf{u}, \mathbf{s}, \mathbf{z})=\mathbf{A}_{u} \mathbf{u}-\mathbf{b}_{u} \\
& \nabla_{s} F_{\epsilon}(\mathbf{u}, \mathbf{s}, \mathbf{z})=\mathbf{A}_{s} \mathbf{s}-\mathbf{b}_{s} \\
& \nabla_{z} F_{\epsilon}(\mathbf{u}, \mathbf{s}, \mathbf{z})=\mathbf{A}_{z} \mathbf{z}-\mathbf{b}_{z},
\end{aligned}
$$

where the matrices $\mathbf{A}_{u}=\mathbf{A}_{u}(\mathbf{s}, \mathbf{z}), \mathbf{A}_{s}=\mathbf{A}_{s}(\mathbf{u})$ and $\mathbf{A}_{z}=\mathbf{A}_{z}(\mathbf{u})$ are positive definite. Details about $\mathbf{A}$-type matrices and $\mathbf{b}$-type vectors and their expressions are given in (Zanetti et al., 2016). It follows that the minimization of $F_{\epsilon}$ can be addressed via an inexact Gauss-Seidel block scheme or by means of a faster block-coordinate descent algorithm (Zanetti et al., 2016). By setting the gradient equations to zero, the three linear systems can be written:

$$
\begin{aligned}
& \mathbf{A}_{u} \mathbf{u}=\mathbf{b}_{u} \\
& \mathbf{A}_{s} \mathbf{s}=\mathbf{b}_{s} \\
& \mathbf{A}_{z} \mathbf{z}=\mathbf{b}_{z} .
\end{aligned}
$$

From a practical point of view, the stationary point of the objective functional can be found by solving the three linear systems in sequence repeatedly, until small variations of the functional are achieved. More precisely, the method is stopped when the relative difference between the evaluation of $F_{\epsilon}$ on two consecutive iterates $\left(\mathbf{u}^{k}, \mathbf{s}^{k}, \mathbf{z}^{k}\right)$ and $\left(\mathbf{u}^{k+1}, \mathbf{z}^{k+1}, \mathbf{u}^{k+1}\right)$ falls below a given threshold. An energetically convenient initialization of the iterative method is to assign as starting values the values $\mathbf{u}^{0}=\mathbf{g}, \mathbf{s}^{0}=\mathbf{e}$ and $\mathbf{z}^{0}=\mathbf{e}$.

\subsection{The parameters of the functional}

The choice of the functional parameters impacts the properties of the segmentation. The parameters $\mu, \alpha, \beta$ rule the competition between the terms of the functional $F_{\epsilon}$ that takes place during the minimization. Because the parameters remain fixed in the minimization, they can be considered global parameters. The understanding of their impact in very simple cases, such as data containing just one edge or one crease, can help the choice of their values when dealing with more complex datasets presenting similar features. In the following, a local analysis of the expected behavior of a minimizer is given by considering to vary separately each single parameter of the functional. The final characteristics of the solution are the result of the competition between 
all terms which reached a stage of minimal impact on the overall functional energy for a given set of parameter values.

The parameter $\mu$ is related to noise removal. The inverse of $\mu$ represents the scale at which variations in the input data are considered as noise. By increasing the value of $\mu$, the squared distance from $\mathbf{g}$ is penalized, thus the solution $\mathbf{u}$ tends to be very close to $\mathbf{g}$. In practice, only variations at the $1 / \mu$ scale are smoothed. This is not a desirable effect in noise-removal applications for which low values of $\mu$ should be used. On the other hand, in processing synthetic or low-noised images, high values of $\mu$ allow for a high-fidelity description of data features.

The Hessian term is weighted by a unitary coefficient. Intrinsically, the squared norm of the Hessian matrix is a measure of the curvature of the surface. When the relative weight of the Hessian term is high with respect to the other terms (weighted by $\mu, \alpha, \beta$ ), the solution is forced to have a quasi-null Hessian. Because of the coupling between the Hessian norm and the function $\mathbf{z}^{2}$, the points where this linear tendency is not energetically convenient are automatically excluded by letting the function $\mathbf{z}$ equals zero. Thus, the function $u$ is expected to be piecewise nearly-linear.

The integral terms associated to $(\alpha-\beta)$ and $\beta$ converge in measure to the lengths of the sets $S_{u}$ and $S_{u} \cup S_{\nabla u}$, respectively. Thus, the parameters $\alpha$ and $\beta$ control the length of the detected discontinuity sets. In particular, high values of $(\alpha-\beta)$ or $\beta$ penalize their length, so they can be seen as contrast parameters. Notice that, because of the constraint $\beta \leq \alpha \leq 2 \beta$ (that ensures the validity of the $\Gamma$-convergence approximation of (5) to the relaxed functional (4)) the choice of the values $(\alpha-\beta)$ and $\beta$ is not symmetric. In fact, for $\alpha=\beta$ the weight on the length of $S_{u}$ is $\alpha-\beta=0$ and the weight on the length of $S_{u} \cup S_{\nabla u}$ is $\beta$. Whereas, if $\alpha=2 \beta$ the weights are in both cases $\beta$. It follows that the length of $S_{u} \cup S_{\nabla u}$ is penalized at least as much as twice the length of $S_{u}$.

\subsection{The $\Gamma$-convergence parameter $\epsilon$}

Theoretically, the discontinuity sets $S_{u}$ and $S_{u} \cup S_{\nabla u}$ are numerically undetectable as they have null $\mathcal{H}^{2}$-measure. By minimizing the elliptic functional $\mathcal{F}_{\epsilon}$, it is possible to keep track of the discontinuity sets via their tubular neighborhoods. The auxiliary functions $s_{\epsilon}, z_{\epsilon}$ move from 1 to 0 smoothly in a neighborhood of the discontinuity sets and they approach 0 at a rate that depends on the geometrical properties of the input image $g$. The width of the tubular neighborhoods of the discontinuity sets shrinks as the $\Gamma$-convergence 
parameter $\epsilon$ tends to 0 . It follows that in a discrete setting the underlying grid must be fine enough to resolve the geometry of the functions $s_{\epsilon}, z_{\epsilon}$ (approximated by the discrete functions $\mathbf{s}, \mathbf{z}$, respectively). Conversely, given a fixed grid size (determined by the parameters $t_{x}, t_{y}$ ), the parameter $\epsilon$ must be chosen in such a way that the resulting grid can detect the neighborhood of the discontinuity sets. Given the nature of the discrete differential operators used in (6) for approximating $\nabla(\cdot)$ and $\nabla^{2}(\cdot)$ (forward differences for first partial derivative and central differences for second partial derivative), an optimal detection of the discontinuity sets corresponds to a choice of $\epsilon$ such that edges are detected along 1 pixel wide curves and edges and creases are detected along 2 pixel wide curves.

\subsection{The 1D Blake-Zisserman model on closed curves}

Following Benciolini and Vitti (2015), the one-dimensional BZ model is presented and applied for recovering polygonal shapes by means of closed curve fitting of noisy observations. Theoretical results and a first implementation of the Blake-Zisserman model in one dimension have been given by Bellettini and Coscia (1994a,b), whereas some applications of the 1D BZ model have been presented in (Vitti, 2012a; Borghi et al., 2012). In this paper, the approach that exploits the numerical formulation given in (Zanetti and Bruzzone, 2017) is adopted for the 1D case and periodic conditions are considered.

Let $\Phi=\left(\varphi_{1}(t), \varphi_{2}(t)\right) \subset \Omega \subset \mathbb{R}^{2}$ be a planar curve dependent on the parameter $t \in \mathbb{R}$. The curve is assumed to be closed and piecewise linear (a polygonal shape), thus the parametric functions $\varphi_{h}, h=1,2$ are piecewise linear and periodic. Let us assume that the original structure of the curve $\Phi$ is lost during the discretization of the domain $\Omega$ and the only trace of the curve is a set of points $p_{i}=\left(x_{i}, y_{i}\right), i=1, \ldots, m$ lying on a regular grid (cfr. Figure 2). The functions $\varphi_{h}$ can be reconstructed using the discrete observations $p_{i}$ and exploiting the 1D BZ model with boundary periodic conditions. The choice of periodic conditions avoids the need to handle start and end points in a special manner. The corners of the polygonal shape defined by $\Phi$ can be identified in correspondence to the values $t$ where the gradients of $\varphi_{1}$ and $\varphi_{2}$ are discontinuous. Since the functions $\varphi_{h}$ are never 
discontinuous a reduced version of the BZ functional is considered

$$
\begin{aligned}
\mathcal{F}_{\epsilon}(u, z) & :=\mu \int_{I}|u-g|^{2} d t+\int_{I} z^{2}\left|u^{\prime \prime}\right|^{2} d t+ \\
& +\beta \int_{I}\left[\epsilon|\nabla z|^{2}+\frac{1}{4 \epsilon}(z-1)^{2}\right] d t
\end{aligned}
$$

where $I \in \mathbb{R}$ and $g: I \rightarrow \mathbb{R}$ is the input curve. In a discrete setting, the input curve is represented by the coordinates of the points $p_{i}$. Thus, the discrete functional is implemented as

$$
\begin{aligned}
F_{\epsilon, h}(\mathbf{u}, \mathbf{z})= & \mu\left(\mathbf{u}-\mathbf{g}_{h}\right)^{T}\left(\mathbf{u}-\mathbf{g}_{h}\right)+ \\
& +\left(\mathbf{D}_{t t} \mathbf{u}\right)^{T} \mathbf{M}_{\mathbf{z}^{2}}\left(\mathbf{D}_{t t} \mathbf{u}\right)+\beta\left\{\epsilon\left(\mathbf{D}_{t} \mathbf{z}\right)^{T}\left(\mathbf{D}_{t} \mathbf{z}\right)+\frac{1}{4 \epsilon}(\mathbf{z}-\mathbf{e})^{T}(\mathbf{z}-\mathbf{e})\right\}
\end{aligned}
$$

where $\mathbf{g}_{1}=\left(x_{1}, \ldots, x_{m}\right)^{T}$, when solving for $\varphi_{1}$, and $\mathbf{g}_{2}=\left(y_{1}, \ldots, y_{m}\right)^{T}$, when solving for $\varphi_{2}$. The discrete operators for first and second order derivatives are defined taking into account periodic conditions by setting:

$$
\mathbf{D}_{t}:=\left(\begin{array}{ccccc}
-1 & 1 & & & \\
& -1 & 1 & & \\
& & \ddots & \ddots & \\
& & & -1 & 1 \\
1 & & & & -1
\end{array}\right), \quad \mathbf{D}_{t t}:=\left(\begin{array}{ccccc}
-2 & 1 & & & 1 \\
1 & -2 & 1 & & \\
& \ddots & \ddots & \ddots & \\
& & 1 & -2 & 1 \\
1 & & & 1 & -2
\end{array}\right)
$$

Similarly to the case of the 2D implementation (cfr. Section 2.2) the functional (11) is quadratic with respect to $\mathbf{u}$ and $\mathbf{z}$. Therefore, an iterative method based on gradient equations is used to address the minimization. Similar considerations can also be made for the selection of the functional parameters and the initialization of the method, as previously done.

Given a minimizing couple $\left(\mathbf{u}_{\epsilon}, \mathbf{z}_{\epsilon}\right)$ of the functional (11), the function $\mathbf{u}_{\epsilon}$ turns out to be the reconstruction of the parametric function $\varphi_{i}$, and $\mathbf{z}_{\epsilon}$ is the indicator function of its gradient-discontinuity points.

\subsection{Remarks about the use of the BZ model in the problem at hand}

After describing the mathematical models of MS and BZ, it can now be seen that the BZ functional is a very well suited mathematical tool for the detection of roof planes and building footprints. The general advantages of BZ over MS has already been mentioned; here the fact that the BZ model 
is suited for our purpose is further addressed. In fact, roof planes can have a significant slope, with a variation of elevation that can be comparable to the planimetric size of the planar patch itself. As a consequence a smoothing term based on the gradient, as the one in the MS functional, would be totally inappropriate. On the other hand the smoothing term based on the Hessian, as the one in the BZ functional, allows to meet the requirement on the planarity of roof elements under investigation. The separation between roof planes is marked by the different orientation of the elements, therefore it results in a discontinuity of the gradient of the elevation. Eave lines, and hence building boundaries, correspond to a localized variation (discontinuity) of the value of the elevation. This means that the three unknown functions of the BZ model involved in the proposed procedure are the natural candidates for detection of roof planes and building footprints. Moreover, the same model is equally suitable for the detection of corners in plane curves and for fitting points on the plane by means of a piecewise smooth curve.

Point clouds and regular grids are both discrete data because of the survey and processing techniques involved in their production. Both of them are used as representations of real objects. A precise definition of edges and corners can be made only on the continuous setup. Despite the discrete character of data, it is possible to distinguish variations between data values at close points that are low enough to be modeled by means of continuous functions from variations that can be considered (i.e. modeled) as discontinuities. In this work, from the analytical point of view, edges and corners are the sets of those points where in the minimization of the BZ model it is less costly to introduce a discontinuity in the approximating function $u$ than to smooth the same sets of points. From the practical point of view, edges and corners are the sets of those grid points where the values of the discrete approximations of the discontinuity functions $s$ and $z$ are close to zero. The sets of those points are considered the representation of features of real objects that, according to the task of this work, are buildings, roof planes and polygons. It is clear that the extraction of the various features mentioned requires some kind of filtering.

\section{The proposed procedure for roof planes detection}

In order to achieve the detection of roof planes in gridded height data a specific procedure has been defined. The procedure involves a sequential application of the BZ model (in 2D and 1D) and can be divided into four 
tasks that are outlined in Figure 4. Task 1 is concerned with the application of the 2D BZ segmentation on an input DSM. The outputs of this block are fundamental for further processing. In Task $\mathbf{2}$ the edge map is processed and building footprints are extracted and labeled. Filtering is based on both the distribution of the normals to the boundary and the size of the footprints. Normals are calculated over an approximation of the boundary given by the 1D BZ. In Task 3 roof planes related to each building footprint are detected via the edge\&crease map and the planar model of each patch is computed using the TLS method on the piecewise smooth approximation of the input data. Lastly, in Task 4 each roof plane is refined by adding points close to the boundary by exploiting the TLS planes and a point-test procedure based on a proximity measure. The outline of the procedure is given in Figure 4. The other figures of this section have been produced applying the procedure to a $1 \mathrm{~m}$ spatial resolution DSM provided by the Provincia Autonoma di Trento $^{1}$ (Italy).

\footnotetext{
${ }^{1}$ http://www.territorio.provincia.tn.it
} 


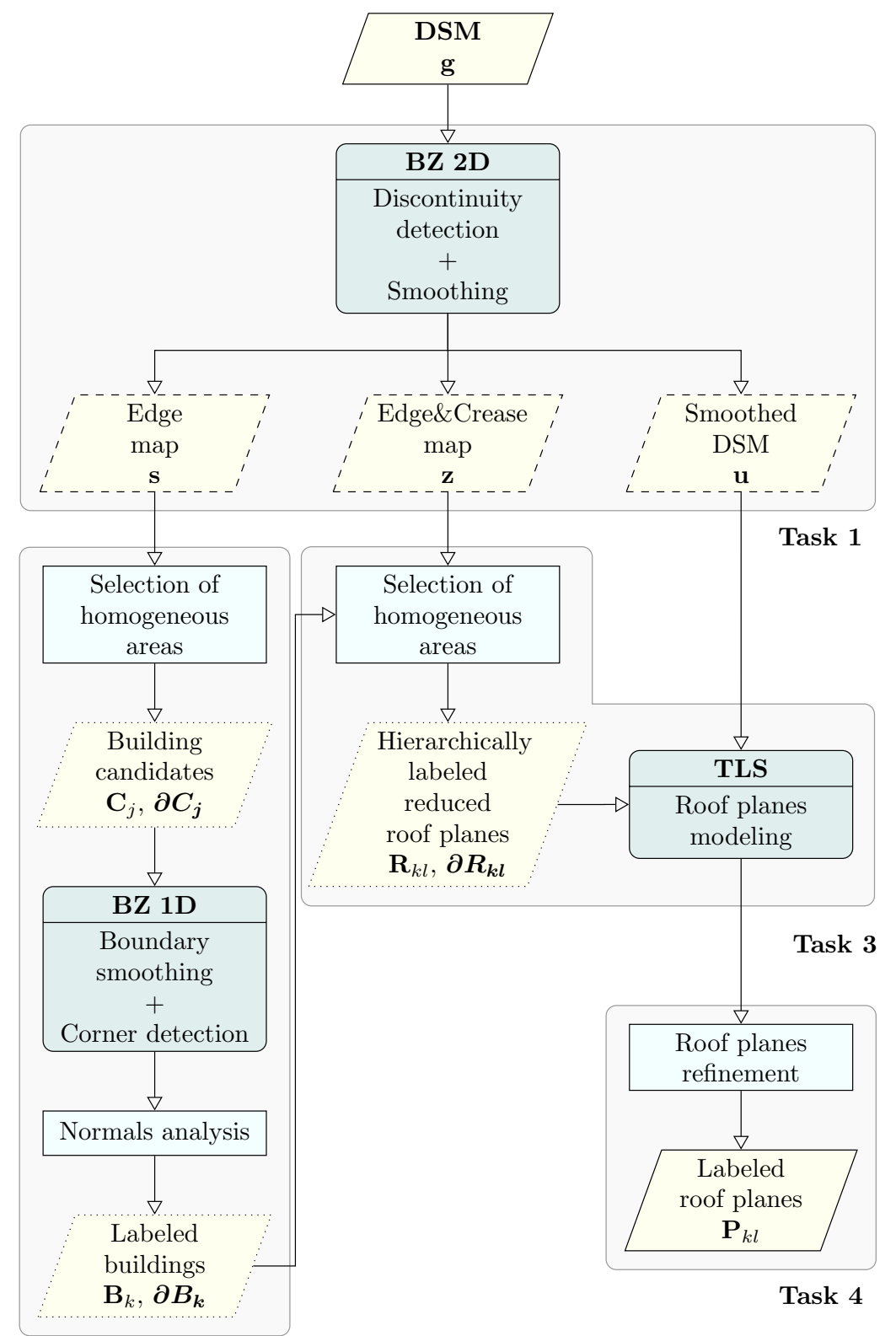

Task 2

Figure 4: Block scheme of the proposed procedure. 


\section{Task $1-2 \mathrm{D}$ variational segmentation}

The key point in the proposed procedure is that the 2D BZ model is able to produce a piecewise nearly-linear approximation $\mathbf{u}$ of $\mathbf{g}$ (being $\mathbf{g}$ the input DSM), and the edge and edge\&crease maps, $\mathbf{s}$ and $\mathbf{z}$ respectively. An example of the BZ outputs is shown in Figure 5. Details about the implementation of the segmentation algorithm are given in Section 2.2. As discussed in Section 2.4 , the discontinuity functions $\mathbf{s}$ and $\mathbf{z}$ are smooth functions taking the value 1 almost everywhere on the domain $\Omega$ and the value 0 where discontinuities are detected. However, the transition between 0 and 1 is not sharp but smooth, so it may happen that the grid resolution is fine enough to let the discrete functions $\mathbf{s}$ and $\mathbf{z}$ to assume values between 0 and 1 in proximity of detected discontinuities. Thus, a simple thresholding of the functions is used to make $\mathbf{s}$ and $\mathbf{z}$ binary images with only 0 and 1 values.

\section{Task 2 - Building detection via 1D variational segmentation}

Following the ideas presented in Section 1.3, building footprints can be extracted as connected regions with a closed boundary made of edge points, i.e. grid points $(i, j)$ such that $\mathbf{s}(i, j)=0$.

Identification of building candidates. Firstly, a 4-connectivity connected components detection algorithm is applied to the binary image $\mathbf{s}$ to detect connected regions. The outcome of the algorithm is thus the set of all building footprint candidates. Let us denote by $C_{j}, j=1, \ldots, m$, the detected regions. Boundary points of each connected region are denoted by $\partial C_{j}$.

Small candidates are not likely to be buildings. A size filtering step is based on the elimination of small objects. This can be done by considering only those $C_{j}$ with an area greater than a given threshold $a_{m i n}$. A similar approach is used to filter out the ground.

Boundary normals filtering. In order to distinguish between candidates with a polygonal footprint (likely to be buildings) and those with an irregular boundary (objects not representing a building), the distribution of the directions of the normals of the candidate boundaries are studied. Since the data domain is discrete, the values of the normal directions computed on the points of a boundary are affected by the grid structure, see Figure 6 (also cfr. Figure 2 in Section 1.3). To overcome this issue, the 1D BZ is applied to approximate the boundary points of a given building candidate (see details of implementation in Section 2.5). The normal directions are hence computed for a smooth boundary with well preserved corners. The modulo operation is 


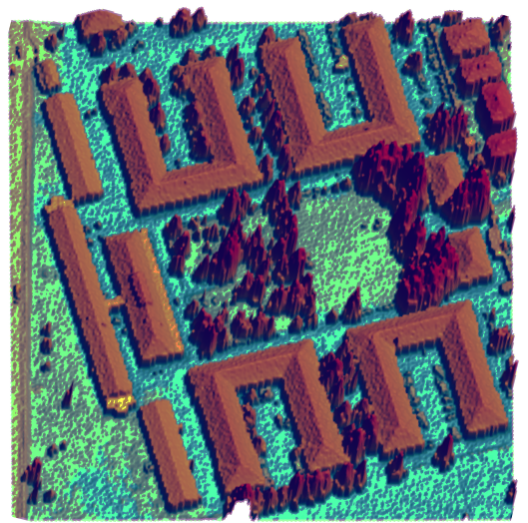

(a)

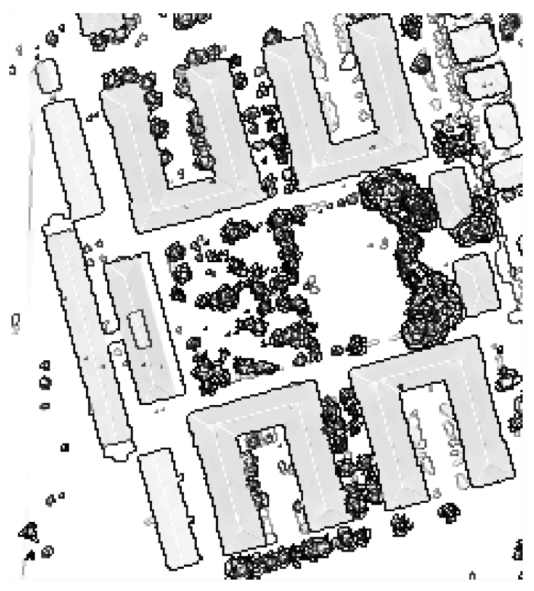

(c)

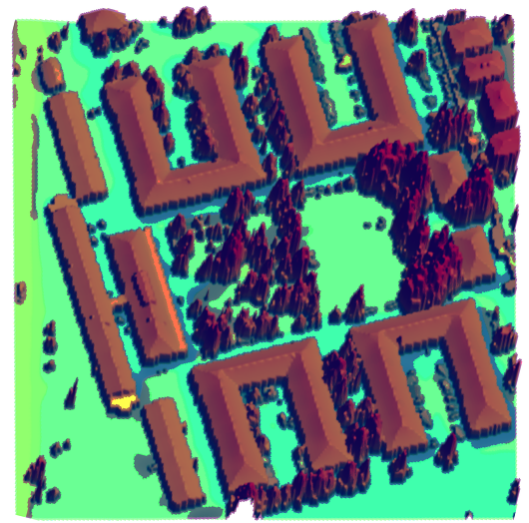

(b)

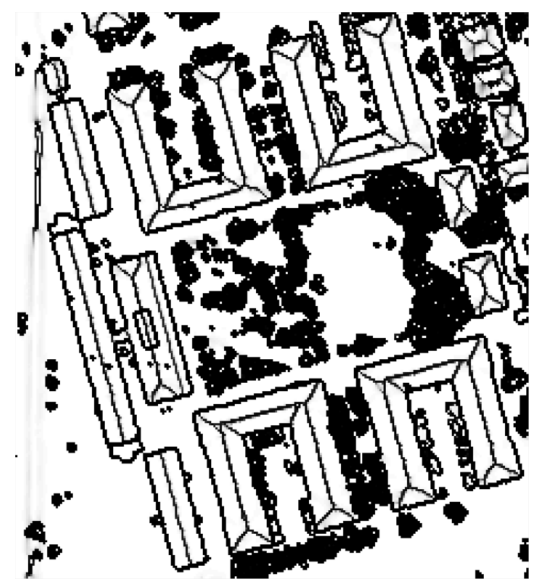

(d)

Figure 5: An example of the outcome of Task 1: (a) Input DSM; (b) the piecewise nearlylinear approximation $\mathbf{u}$ of $\mathbf{g}$; (c) the edge map $\mathbf{s}$; (d) the edge\&crease map $\mathbf{z}$.

then applied to compute the reminder of the division of the angular direction of the boundary normals by $\pi / 2$. By observing that the output values are very little scattered in case of polygonal shapes built of linear arcs forming just right angles, buildings are selected by filtering the returns of the modulo operator.

After area and boundary normals filtering, the initial set of connected regions is reduced to a subset that for notation convenience is denoted by $B_{k}, k=1, \ldots, n$. The family $B_{k}$ is the set of detected building footprints and $\partial B_{k}$ are their boundaries. 


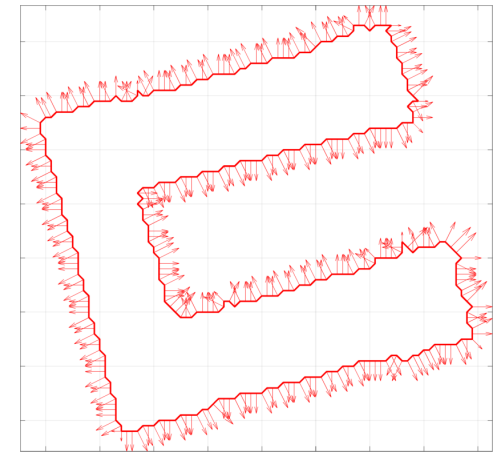

(a)

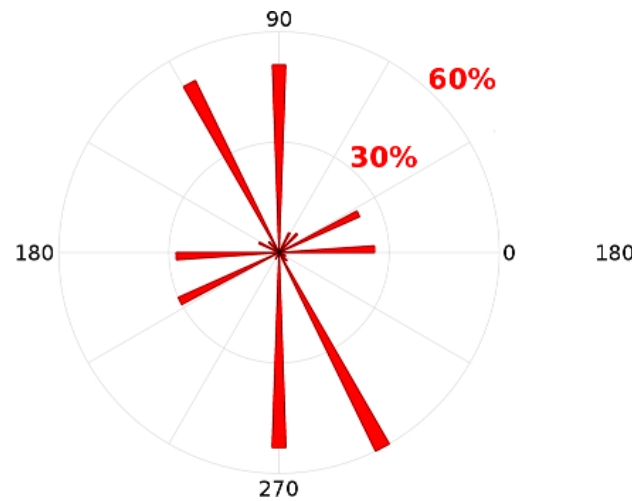

(c)

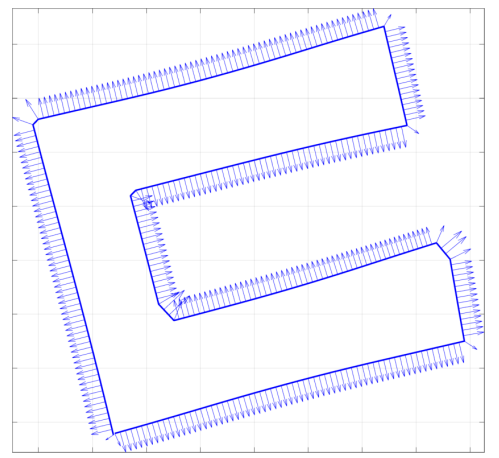

(b)

90

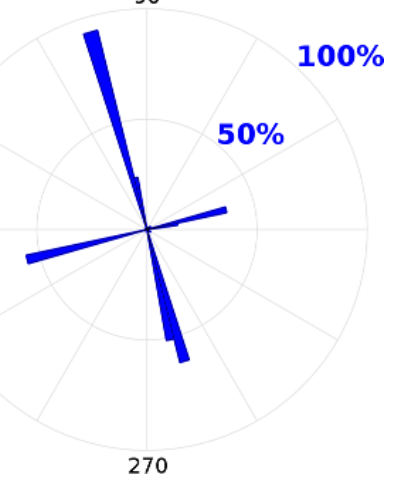

Figure 6: An example of building shape regularization with BZ-1D: (a) detected boundary with normals; (b) regularized boundary with normals; (c) circular histograms of the normals in both cases, but with different radius scales.

\section{Task 3 - Roof planes detection and modeling}

Again, following the discussion in Section 1.3, in correspondence to any building footprint $B_{k}$ it is now possible to exploit the edge\&crease function $\mathbf{z}$ to detect roof plane boundaries related to the building.

Hierarchical detection of roof planes. By running a connected components detection algorithm on the binary function $\mathbf{z}$ for every single $B_{k}$, a set of $n_{k}$ components $R_{k l}, l=1, \ldots, n_{k}$ is obtained. The elements $R_{k l}$ correspond to the roof planes of the building $B_{k}$. Similarly to the case of building footprints the boundaries of the region $R_{k l}$ are denoted by $\partial R_{k l}$. Notice that $R_{k l} \subset B_{k}$ for all $l=1, \ldots, n_{k}$, but boundary points are not assigned to any roof plane, thus such $R_{k l}$ are referred to as reduced roof planes. In Figure 7, images (1) 
and (2) show an example of candidate buildings and of hierarchical reduced roof planes.

Roof plane modeling via TLS. Each $R_{k l}$ is thus a set of points locating a quasi-planar patch of the function $\mathbf{u}$. A TLS approximation of this patch can be computed by studying the empirical covariance matrix $M_{k l}$ of the point coordinates. Let $\lambda_{k l}:=\min \left\{\lambda: \lambda\right.$ eigenvalue of $\left.M_{k l}\right\}$ and $\mathbf{n}_{k l}$ its corresponding unit eigenvector. Then, $\mathbf{n}_{k l}$ is the unit vector normal to the plane of best least squares approximation; with the equation of the plane being

$$
\mathbf{n}_{k l} \cdot \mathbf{x}-\mathbf{b}_{k l}=0
$$

where $\mathbf{b}_{k l}=\mathbf{n}_{k l} \cdot \overline{\mathbf{x}}_{k l}$ and $\overline{\mathbf{x}}_{k l}$ is the mean of the $3 \mathrm{D}$ points $\mathbf{x}$, and $\lambda_{k l}$ is also the variance of the distances of the $3 \mathrm{D}$ points with respect to the TLS plane given by (12). The term $\mathbf{b}_{k l}$ is the displacement of such plane with respect to the origin of the coordinate system. The parameters of the fitting planes are supposed to be estimated from a uniformly large number of points.

\section{Task 4 - Roof planes refinement}

So far, the points belonging to $\partial B_{k}$ or $\partial R_{k l}$ have not yet been assigned to any roof patch. When proper values of the functional parameters are selected, edges will be located along 1 pixel wide curves (because the gradient operator is approximated by using forward differences), whereas the edge\&crease points will be located along 2 pixel wide curves (as the Hessian operator is approximated using 2 nd order central differences). Wider traces are also possible, essentially because of the interaction between the $\Gamma$-convergence parameter $\epsilon$ and the grid size, see (Vitti, 2012b) and (Zanetti et al., 2016). However, given a discontinuity point, the decision of which adjacent roof patch the point belongs to can not be done in an automatic way unless the discontinuity line lies right along the $x$ or the $y$ direction.

Boundary points test. The planar models corresponding to the components $R_{k l}$ can be further refined by testing all points that are close to their boundaries. In order to do this, firstly each component $R_{k l}$ is enlarged using morphological dilation with a disk structuring element of radius 2 . Note that using radius 1 the dilation of $R_{k l}$ coincides with the union of $R_{k l}$ and $\partial R_{k l}$. The choice of radius 2 ensures that all neighboring points of the patch are considered along with uncertain edge pixels. Moreover, within each building footprint the dilated components can be mutually intersected in order to determine their adjacency. Let us denote $\bar{R}_{k l}$ the dilated component. The refinement procedure consists of two steps: 
1. any grid point $p_{i} \in \bar{R}_{k l} \backslash R_{k l}$ is discarded if $\mathbf{x}_{i}$ is not admissible to the plane model given by (12); that is when

$$
\left|\mathbf{n}_{k l} \cdot \mathbf{x}_{i}-\mathbf{b}_{k l}\right|>3 \sigma_{k l}
$$

where $\sigma_{k l}=\lambda_{k l}^{1 / 2}$

2. any grid point $p_{i} \in \bar{R}_{k l}$ is tested against all the patches of the same building $j$ that are close to the current patch $k$; the set of the patches that may be refined by the inclusion of the point $p_{i}$ are named $\bar{R}_{<k l>}$; the point $p_{i} \in \bar{R}_{k l} \cap \bar{R}_{<k l>}$ is hence removed from $\bar{R}_{k l}$ when

$$
\frac{\left|\mathbf{n}_{k l} \cdot \mathbf{x}_{i}-\mathbf{b}_{k l}\right|}{\sigma_{k l}}>\frac{\left|\mathbf{n}_{<k l>} \cdot \mathbf{x}_{i}-\mathbf{b}_{<k l>}\right|}{\sigma_{<k l>}} .
$$

The latter test ensures that each 3D point is assigned to the closest planar patch, where the metric used is the orthogonal distance weighted by the standard deviation of the fitting plane.

With the completion of this last step, the dilated planar patches are filtered and include only compatible boundary points. The family of these patches is the final set of the hierarchically detected refined roof planes, denoted by $P_{k l}$. In Figure 7, image (3) shows an example of detected final roof planes.

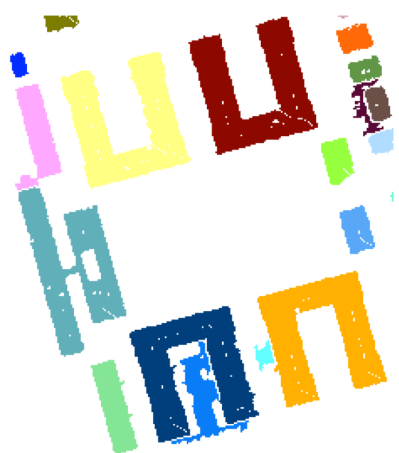

(a)

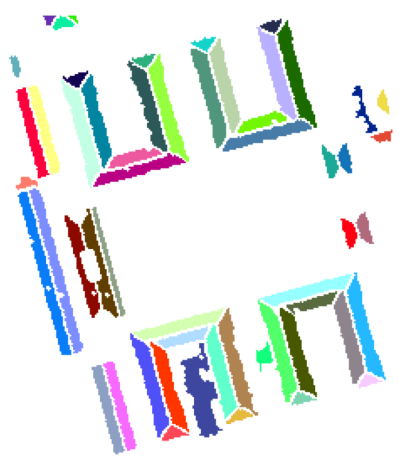

(b)

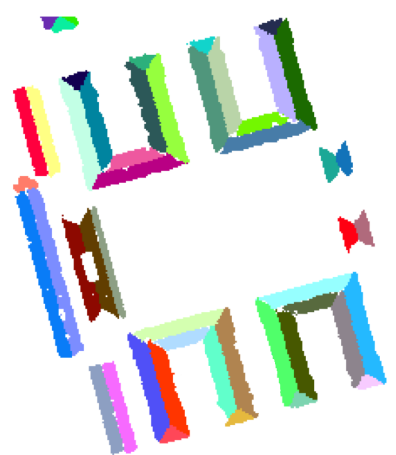

(c)

Figure 7: An example of candidate buildings, hierarchical reduced and final roof planes: (a) candidate buildings (with spurious object not yet filtered out); (b) reduced planar patches; (c) final roof planar patches (after filtering). 


\section{Detailed application and validation}

The procedure presented was applied to one of the DSMs provided in the context of the "ISPRS Test Project on Urban Classification and 3D Building Reconstruction" ${ }^{\prime 2}$ (Cramer, 2010). In particular the DSM of Vaihingen Area $3,0.25 \mathrm{~m}$ spatial resolution, was processed. In the following the procedure is briefly recalled and accompanied by a set of figures. Several figures are provided and commented to show intermediate and final results.

Figure 8 presents on the top the input DSM and the orthophoto of the study area (in false colors); on the bottom the corresponding edge and edge\&crease maps, i.e. the outputs of Task 1. The edge map is plotted using and equalized color table to sharpen the richness of information stored in the edge map. It is possible to observe in black the loci where the functions $\mathbf{s}$ and $\mathbf{z}$ reach the value 0 . The regions of white and light-gray pixels, where the functions present values close to 1 , correspond to the DSM regions of homogeneous heights. The Figure shows that the edge map presents features that can be exploited for the detection of building footprints and that the edge\&crease map presents features that can be exploited for the detection of roof planes. In fact, roof ridges are clearly distinguishable.

Figure $9(\mathrm{a})$ presents in black the values $\mathbf{s} \in[0,0.8)$ corresponding to the edge points; in a blue-scale palette the values $\mathbf{s} \in[0,8,0.99]$, corresponding to mildly varying heights and in white the values $\mathbf{s} \in(0.99,1]$ corresponding to the highest homogeneous DSM regions. The image helps to visualize which features of the edge map can be exploited for the detection of building candidates.

Figure 9(b) illustrates the connected regions obtained from the edge map; in the image a binary edge map obtained by applying a threshold of 0.8 to the edge map is overlaid. Connected regions with values $\mathbf{s} \in[0,0.8)$ are in black and regions with values $\mathbf{s} \in(0.99,1]$ are in white. The region representing the ground was removed via area thresholding and it is shown in white. Connected regions with values $\mathbf{s} \in[0.8,0.99]$ are colored according to the unique label assigned. It is possible to observe that some objects, mainly gardens, have not been isolated from the ground. This is due to the fact that somewhere in (the smooth approximation of the) DSM, height variations were not large enough to force the discontinuity function $\mathbf{s}$ to assume values lower than 0.8, thus the edges of these objects are not closed. Figure 9(c)

\footnotetext{
${ }^{2}$ http://www.ifp.uni-stuttgart.de/dgpf/DKEP-Allg.html
} 
shows the building candidates that remain after the removal of candidates with an area smaller than $30 \mathrm{~m}^{2}$. In this latter image the binary edge map is no longer displayed. At this stage the boundaries of the candidate buildings are extracted and passed to the 1D BZ model to produce a piecewise nearlylinear approximation of the boundary with preserved corners. Boundary normals are then computed and analyzed to detect right-angle polygonal shapes. igure $9(\mathrm{~d})$ shows the map of the detected right-angle polygonal buildings, i.e. the output of Task 2 .

Figure 10(a) shows the hierarchical reduced roof planes colored according to the value of the unique label assigned. The edge\&crease map is overlaid to the reduced roof planes map; by comparing Figure 10(a) with Figure 9(b) it is possible to see the roof ridges splitting the building regions permitting the hierarchical labeling of the reduced roof planes. At this stage, every plane is then modeled via TLS and plane boundaries are extracted thus completing Task 3.

Reduced roof planes are then processed according to the steps of Task 4 . Figure 11 shows an example of the refinement step. In this figure, gray dots are points surrounding a given roof; circles in light color represent points assigned to different reduced roof planes; bold dark dots represent the edge points assigned to the closet plane according to the criteria defined in Task 4 . It is possible to distinguish gray dots lying on the small wall that separates the two smaller planes; these dots have not been assigned to any plane, as might have been expected. Figure 12 shows the detail of a single building: the orthophoto and a 3D view of the points of the smoothed DSM are on the top row. In the $3 \mathrm{D}$ view the roof planes are patches in uniform tint and the points surrounding the roof are in gray. The reduced and the final roof planes are shown in the two images on the bottom row of the figure. Final roof planes are shown in Figure 17 for the entire study area.

To describe further the steps of Task 2 that serve to detect right-angle polygonal shapes, two object are considered: a tree and a building. In Figure 13 a set of images are shown to help the description. On the top row, the tree and the building shapes are side by side. Every shape is shown twice: once with boundary normals computed from the piecewise linear curve passing through the center of the boundary pixels and once with boundary normals computed from the BZ approximation of the boundary. On the second row, the polar histograms of the normal directions are shown. The bins are $2^{\circ}$ wide. In Figures 13(e) and 13(g) bins are correctly aligned along only four directions, because of the limited number of possible arrangements 
of three close pixels. On the third row, the returns of the modulo operator are shown, again using polar histograms for both normal types. On the fourth row, the returns of the modulo operator are shown using standard histograms, only for the normals computed from the BZ approximation of the boundary (Figures 13(b) and 13(d)). It is possible to see how the returns of the modulo operator are much more concentrated along one particular value for the polygonal shape than for the tree shape. Figure 14 shows on the left a building shape with its boundary normals (computed from the BZ approximation of the boundary). The two images on the right show the output of the BZ model, i.e. the discontinuity function $\mathbf{s}$ and the approximation $\mathbf{u}$, for the $x$ and $y$ components of the boundary coordinates respectively.

A special search for mixed building-tree objects could be carried out by selecting rejected building candidates that present a high variance of the fitting plane and a sparse distribution of the returns of the modulo operator. Once detected, such kind of objects could be reprocessed with different parameter values. The discontinuity functions $s$ and $z$ could be further exploited as well. The absence of homogeneous areas in the discontinuity function $z$ should help in filtering out the tree part, e.g., see the trees on the upper-right and lower-right corners of Figures 8(c) and 8(d). 


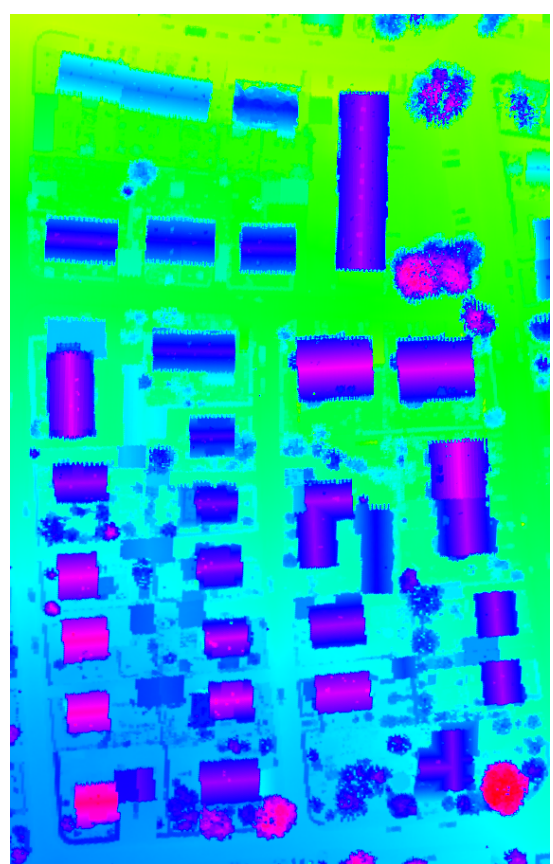

(a)

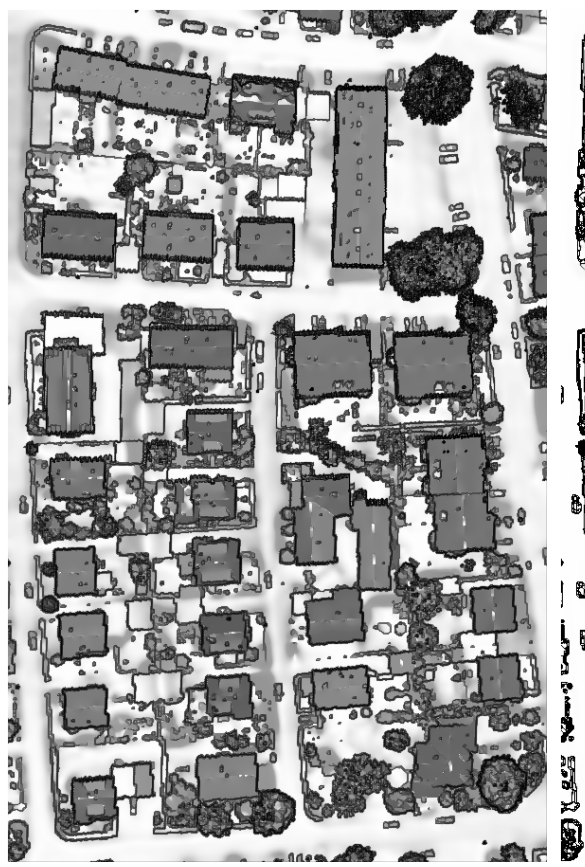

(c)

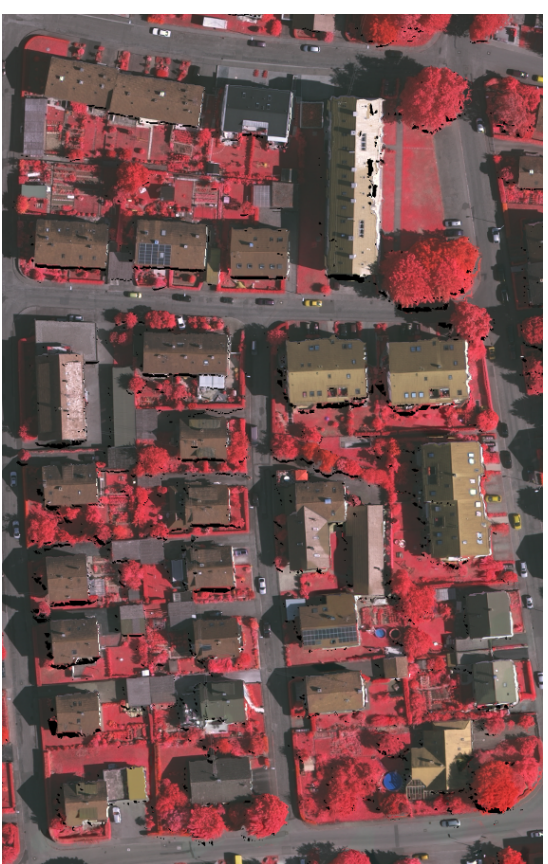

(b)

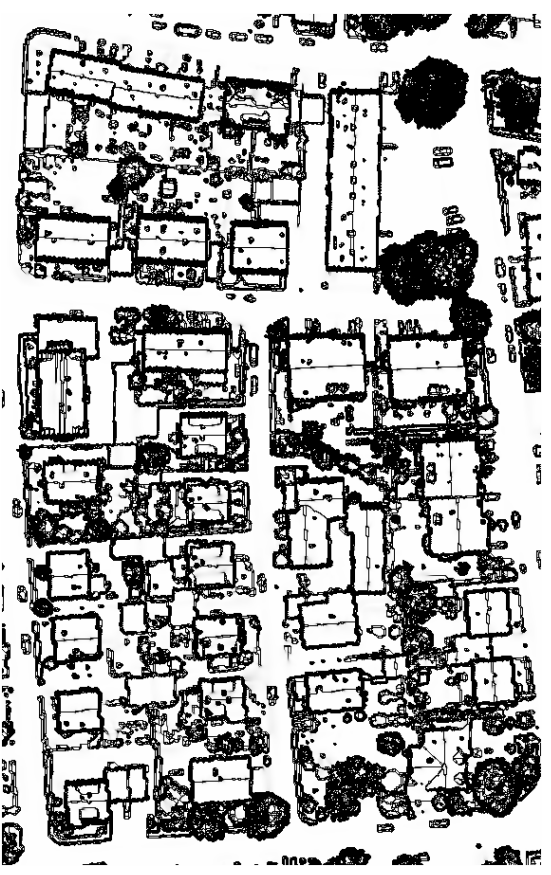

(d)

Figure 8: (a) input DSM); (b) orthophoto in false colors; (c) equalized edge map; (d) edge\&crease map. 


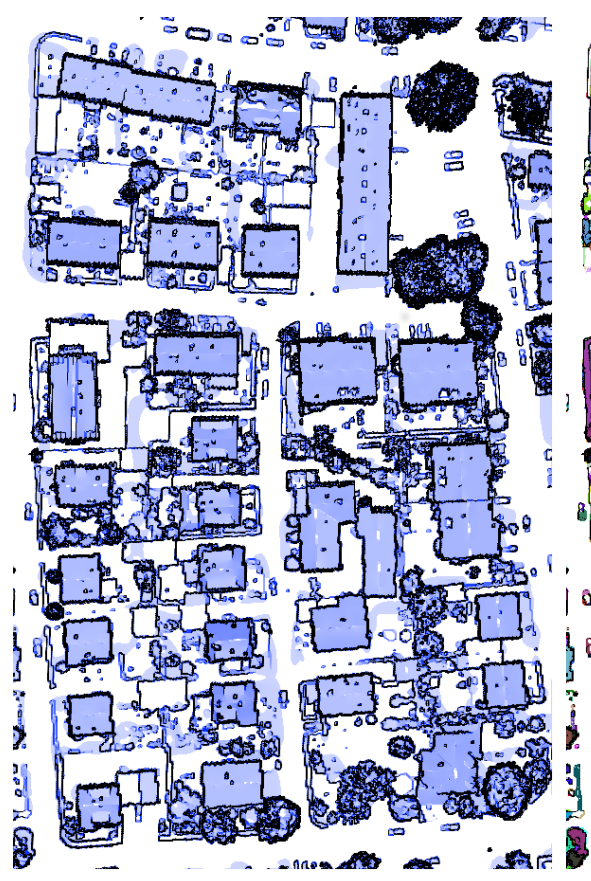

(a)
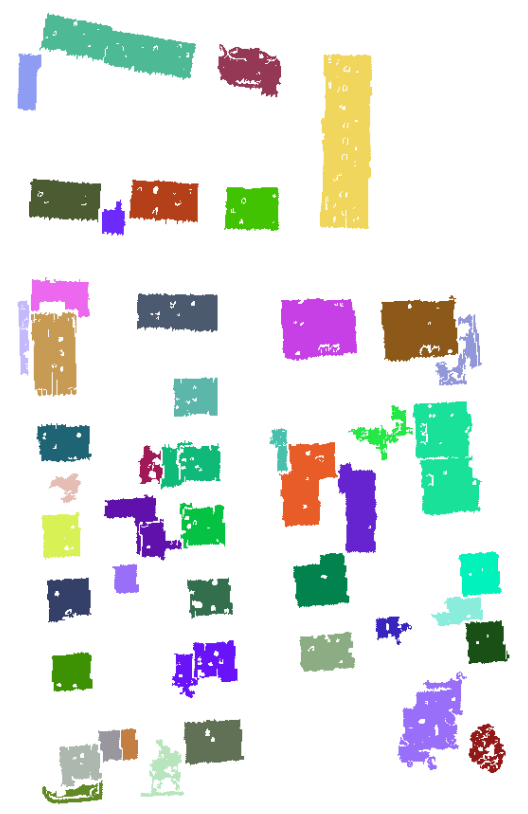

(c)

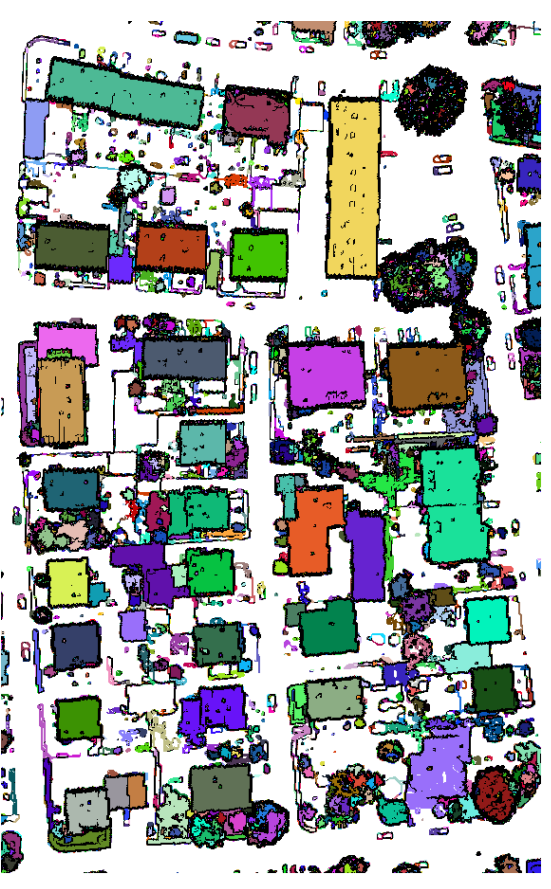

(b)
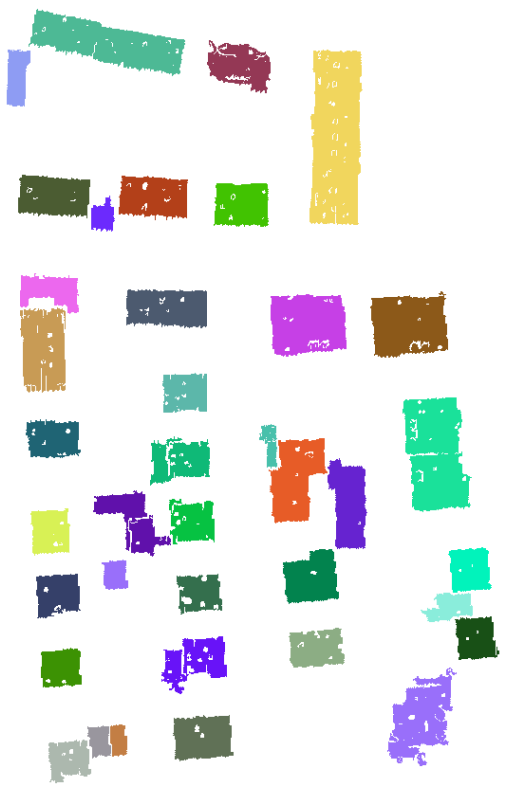

(d)

Figure 9: (a) edge map - values $\mathbf{s} \in[0,0.8)$ in black, values $\mathbf{s} \in[0,8,0.99]$ in light blue, values $\mathbf{s} \in(0,99,1]$ in white; (b) labeled conßsqcted regions with binary edge map overlaid; (c) building candidates; (d) detected buildings. 


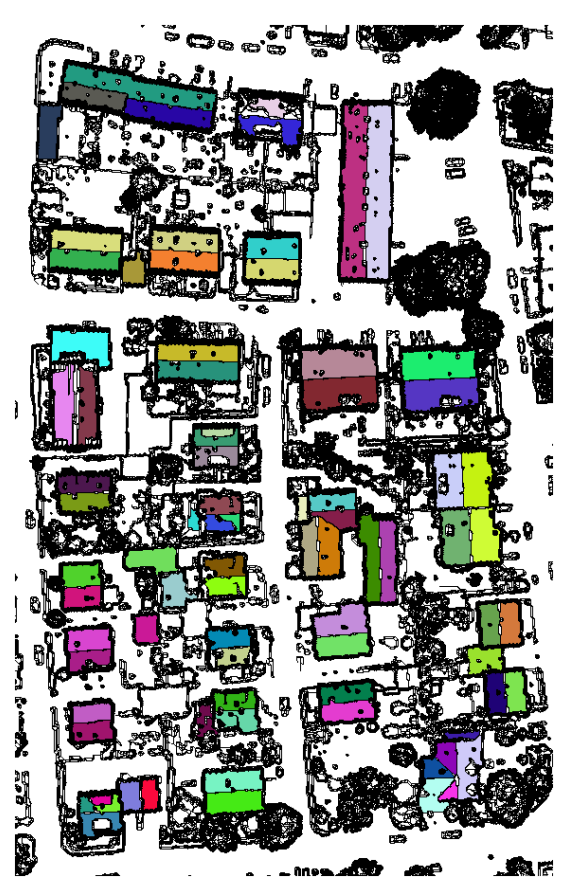

(a)

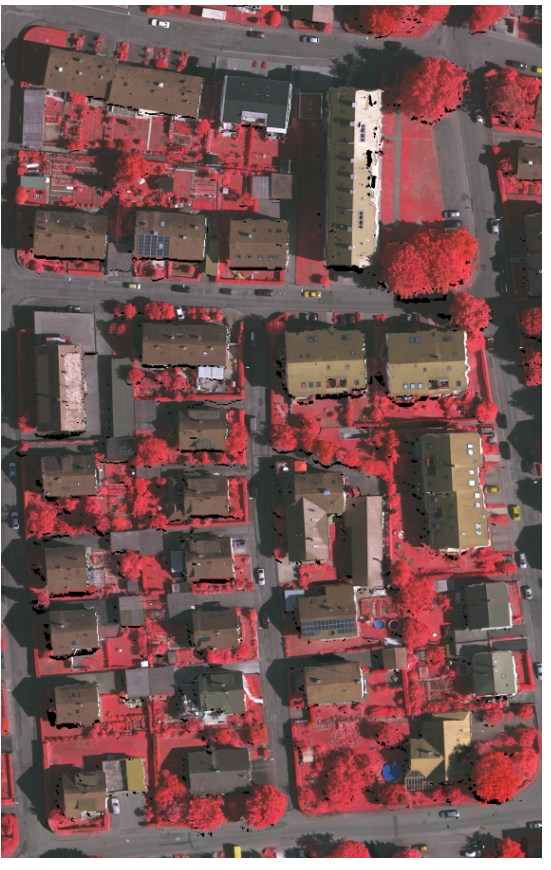

(b)

Figure 10: (a) hierarchical reduced roof planes with edge\&crease map overlaid; (b) orthophoto in false colors.

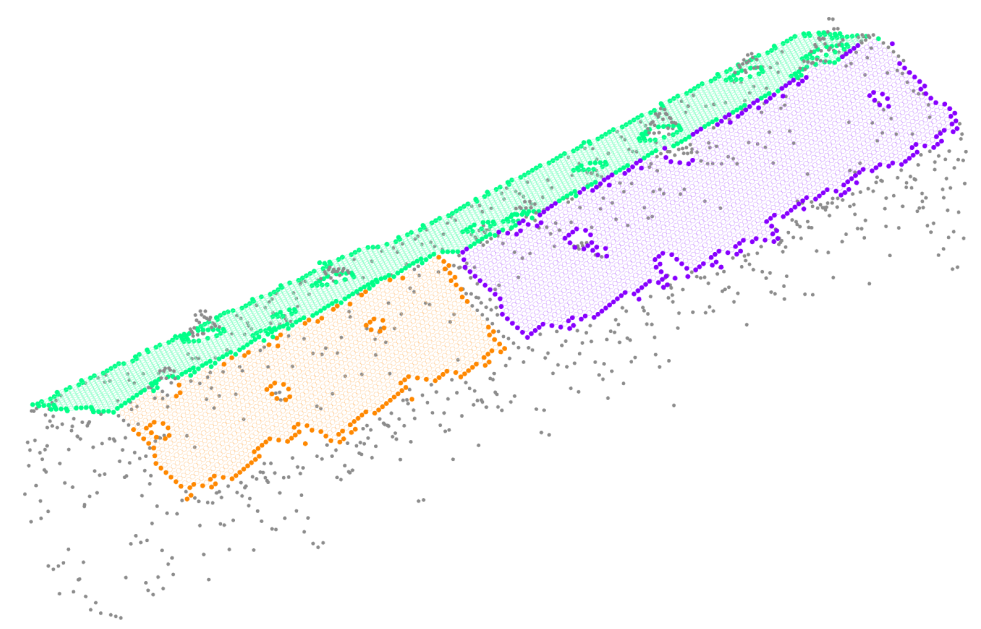

Figure 11: Example of the refinement step. 


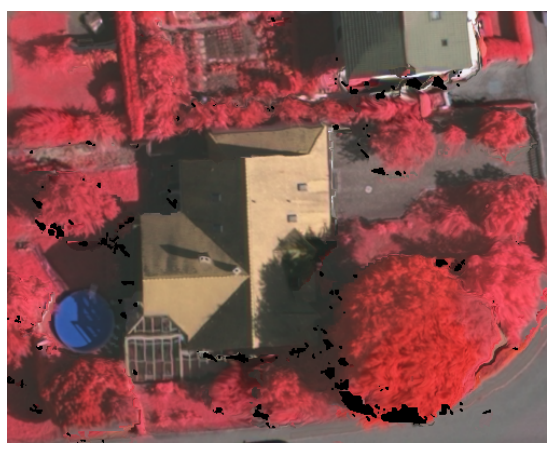

(a)

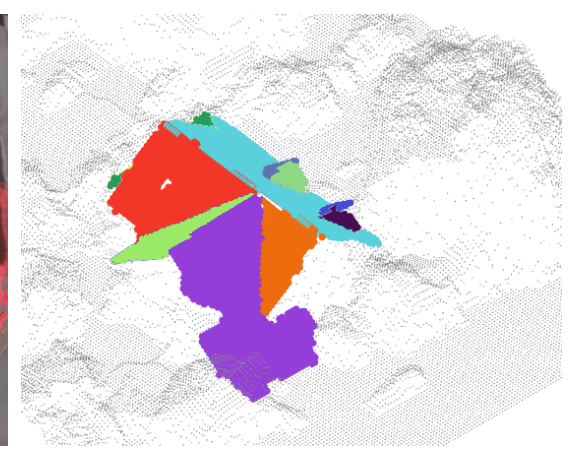

(b)

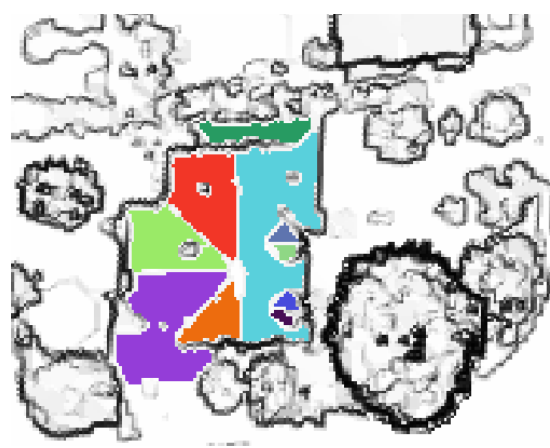

(c)

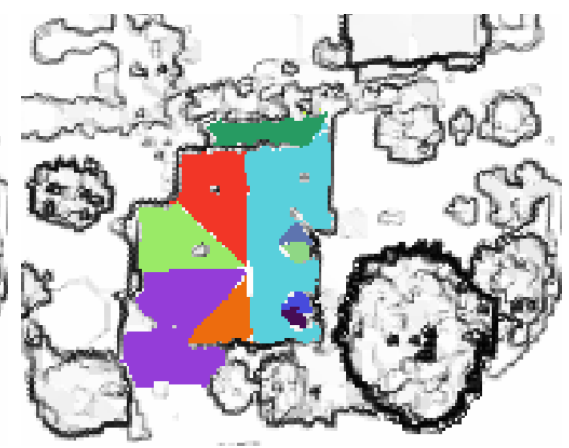

(d)

Figure 12: A single building: (a) orthophoto in false colors; (b) 3D view of smoothed DSM with roof planes; (c) reduced roof planes; (d) final roof planes. 


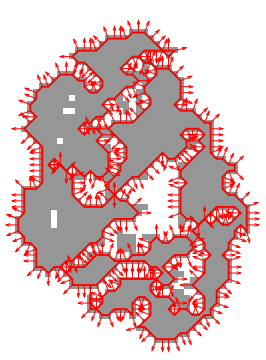

(a)

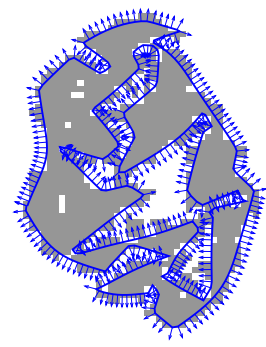

(b)

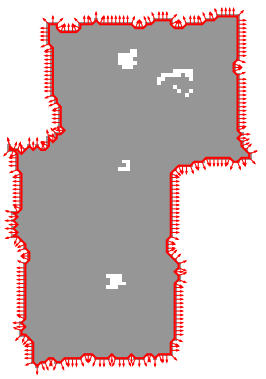

(c)

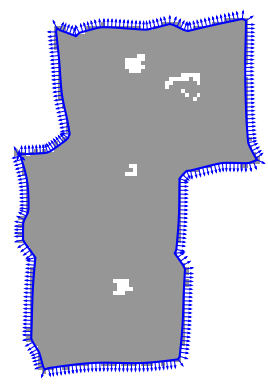

(d)

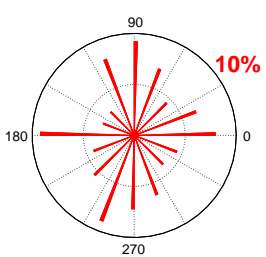

(e)

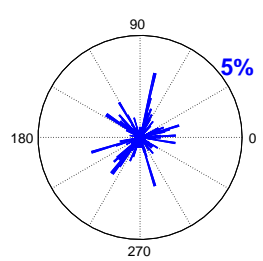

(f)

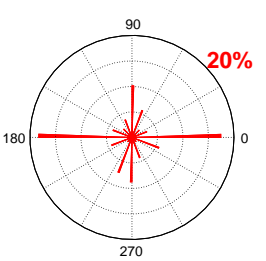

(g)

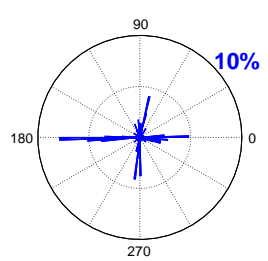

(h)

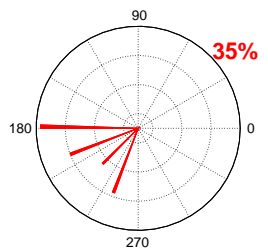

(i)

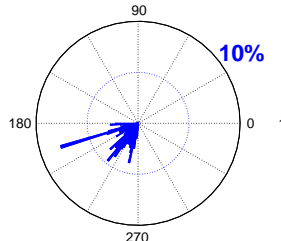

(j)

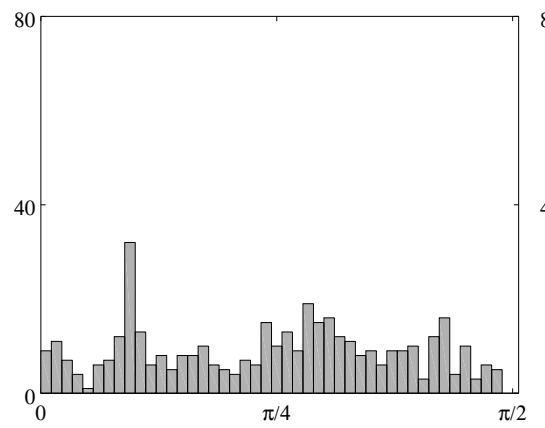

(m)

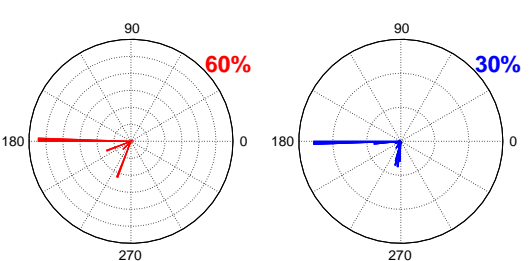

(k)

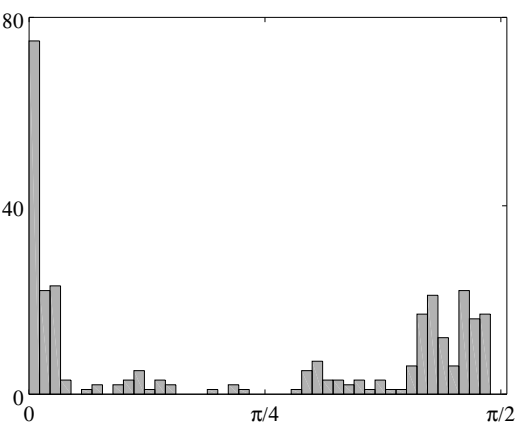

(n)

Figure 13: Boundary normals for the pixel-wise boundary $(\mathrm{a}, \mathrm{c})$ and for the output of the 1D BZ model (b, d) for tree (a, b) and building (c, d) shapes. Polar histograms of the normal directions (e, f, g, h) and of the returns of the modulo operator (i, j, k, l). Histograms $(\mathrm{m}, \mathrm{n})$ of the returns of the modulo operator for the normals obtained from the output of the 1D BZ model (b, d) for tree and building shapes respectively. 


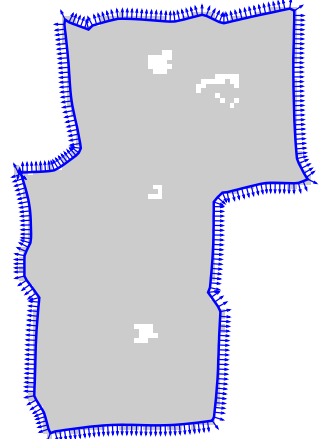

(a)
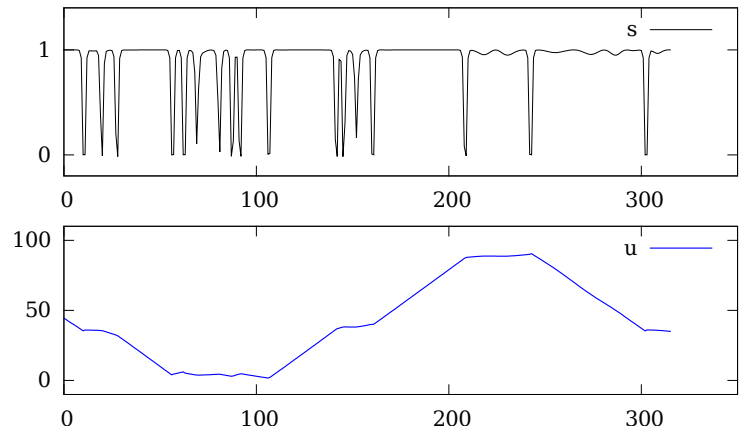

(b)
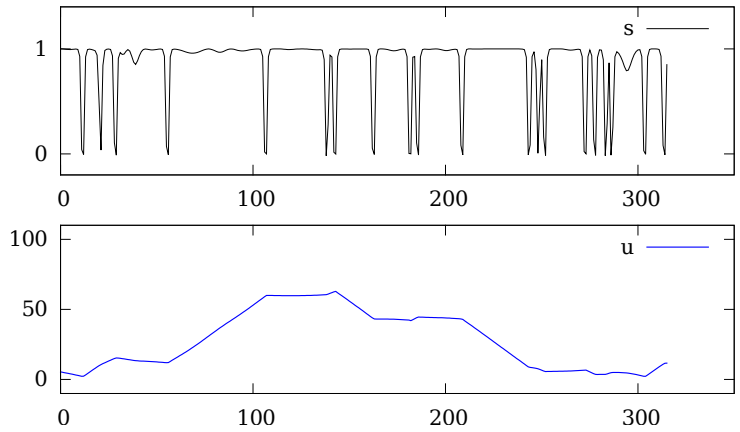

(c)

Figure 14: (a) a building boundary with normals; (b) and (c) output of the 1D BZ model for the $x$ and $y$ components of the boundary coordinates. 


\subsection{Validation}

The results of the application described in Section 4 are evaluated with respect to reference data provided in the framework of the "ISPRS Test Project on Urban Classification and 3D Building Reconstruction". In particular evaluations of thematic accuracy is done on the basis of metrics proposed in (Rutzinger et al., 2009) and adopted in the ISPRS benchmark project (Rottensteiner et al., 2012). Three indices are computed on the base of the count of true positives (TP), false positives (FP) and false negatives (FN), namely:

$$
\begin{aligned}
& \text { completeness }=\frac{\# T P}{\# T P+\# F N} \\
& \text { correctenss }=\frac{\# T P}{\# T P+\# F P} \\
& \text { quality }=\frac{\# T P}{\# T P+\# F N+\# F P} .
\end{aligned}
$$

The indices are computed on a per-object level and on a per-pixel level. In the first case a detected object (a building or a roof plane) is considered a true positive if at least $50 \%$ of its area overlaps an object in the reference data; the quantities FP and FN are evaluated in the same way. In the second case the the number of pixels belonging to the mentioned quantities (TP, FP, FN) is counted. In Figure 15(a) the reference data is shown. Only buildings with an area greater or equal than $30 \mathrm{~m}^{2}$ are considered to be consistent with the threshold adopted in the procedure when the set of building candidates is built. In Figure 15(b) the map of the evaluation of building detection is shown. In the map, true positives are in white, false negatives in dark gray and false positives in black. Values of the metric indices on a per-object level and on a per-pixel level are given in Table 1.

\begin{tabular}{lcc} 
Index & per-object & per-pixel \\
\hline completeness & 0.85 & 0.74 \\
correctness & 0.97 & 0.98 \\
quality & 0.83 & 0.73 \\
\hline
\end{tabular}

Table 1: Per-object and per-pixel metrics.

According to the ISPRS benchmark (Rottensteiner et al., 2012, 2014), a method is considered of practical relevance when the completeness index is greater or equal than 0.70 and the correctness index is greater or equal than 0.85 , on a per-object level. 

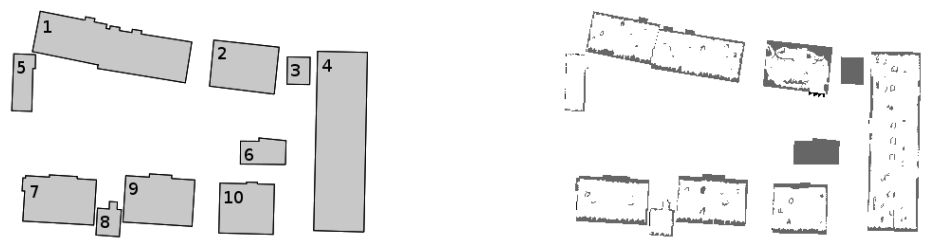

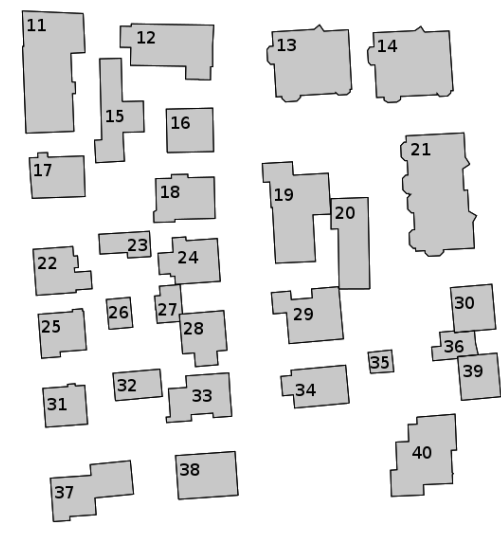

(a)

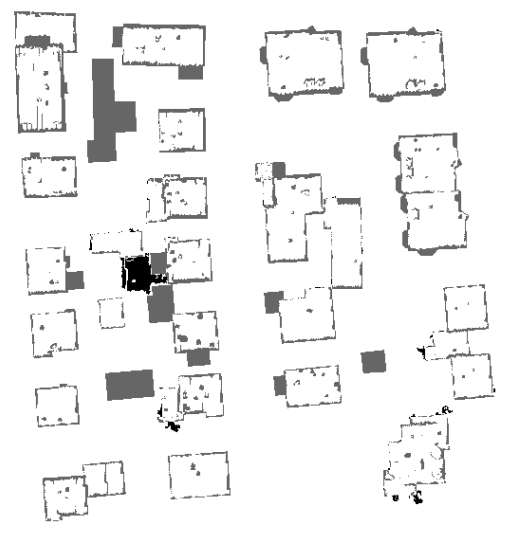

(b)

Figure 15: (a) reference buildings; (b) detected buildings in white, false negative pixels in black, reference buildings in dark gray.

In Figure 15(b) it is possible to see one false positive object. The object is a flat green lawn with a right-angle polygonal shape, surrounded by high shrub-edge objects with regular shape and buildings. False negatives objects, such as objects numbered 3, 6, 15, 27 and 32 in 15(a), are very homogeneous and flat and have been removed from the set of building candidates when the values $\mathbf{s} \in[0,8,0.99]$ was selected, see Figures 9(a) and 9(b). In Figure 16(a) the reference building boundaries are plotted as white lines on the top of the DSM. In Figure 16(b) the detected buildings are also plotted in light gray. The presence of false negative pixels around the borders of detected buildings is due to the presence in the DSM of relevant structures and artifacts near the building walls, e.g. balconies and pixels belonging to the gridded approximation of building walls.

In Figure 17(a) the detected roof planes and the reference map are shown. In Figure 17(b) the map of the evaluation of roof plane detection is shown. In the map, true positives are in yellow, false negatives in blue and false positives 


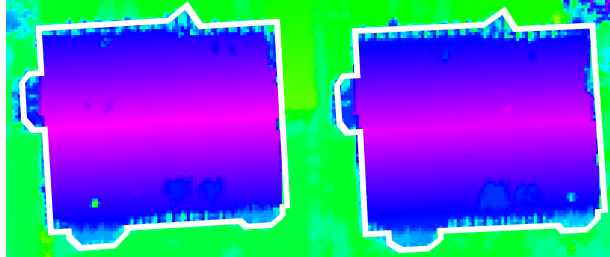

(a)

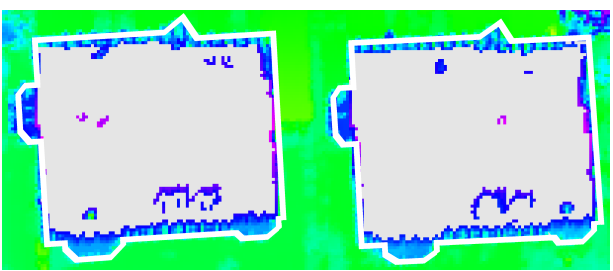

(b)

Figure 16: (a) input DSM with reference building boundaries; (b) input DSM with reference building boundaries and detected building footprints.

in red. On a per-object level, completeness, correctness and quality are 0.81 , 0.98 and 0.80 respectively. On a per-pixel level completeness, correctness and quality are $0.74,0.96$ and 0.72 respectively. False positive and false negative objects in Figure 17(b) are the same of Figure 15(b).

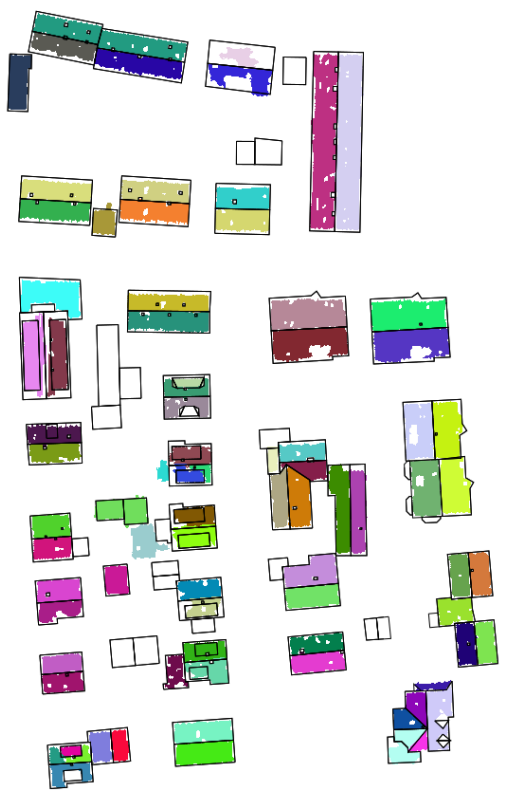

(a)
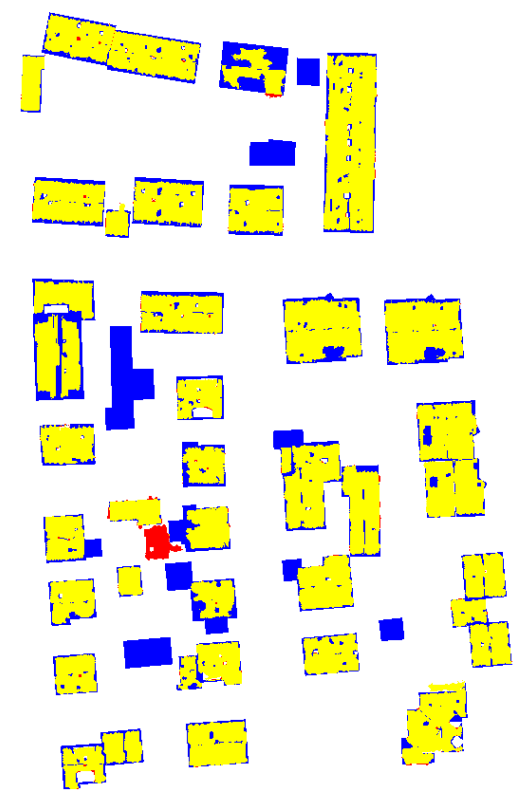

(b)

Figure 17: (a) reference roof planes and detected roof planes; (b) detected roof planes in yellow, false negative pixels in red, reference roof planes in blue. 
Effects of edge preserving data smoothing is illustrated by Figure 18 where the hillshade and the slope maps computed for $g$ and $u$ can be compared. For the set of detected planar patches ( 80 units), the averages of the standard deviations of fitting planes computed from the data $g$ and from the smooth approximation $u$ are $0.221 \mathrm{~m}$ and $0.139 \mathrm{~m}$ respectively. The average of the relative improvement is greater than $40 \%$.

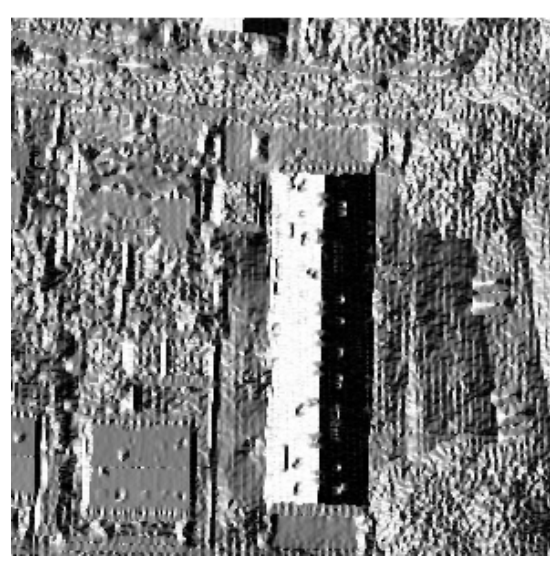

(a)

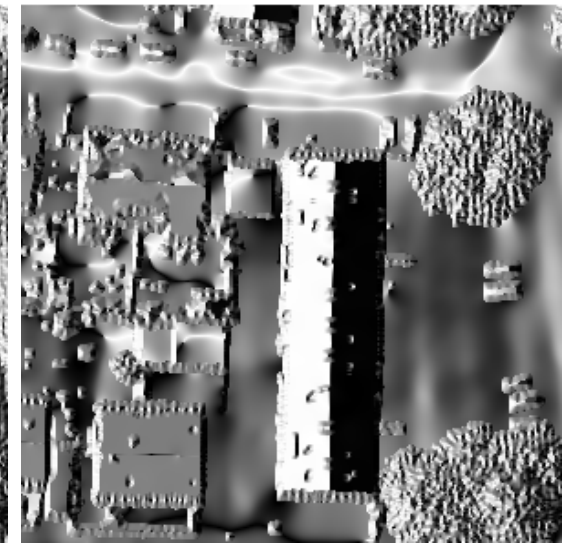

(b)

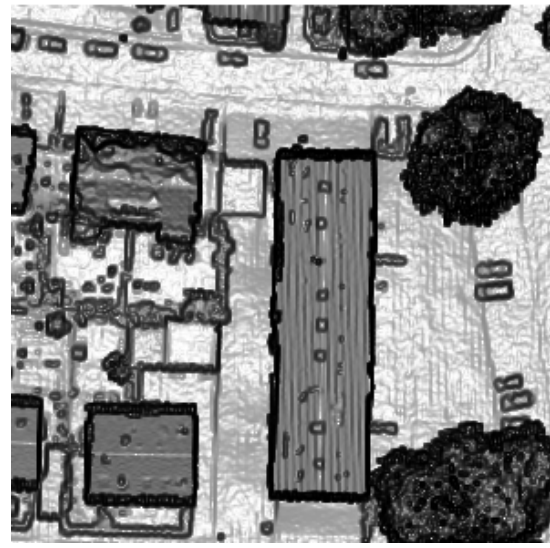

(c)

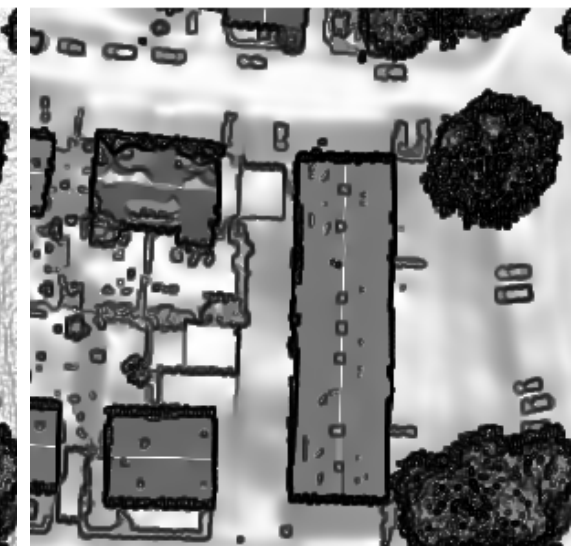

(d)

Figure 18: Impact of edge preserving data smoothing: (a) hillshade map from $g$; (b) hillshade map from $u$; (c) slope map from $g$; (d) slope map from $u$. 


\section{Final remarks and perspectives}

An original procedure for the detection of roof planes is presented. The procedure exploits the BZ 2nd order variational model to obtain a piecewise nearly-linear smooth approximation of the data. At the same time, discontinuities of the approximation and of its gradient are directly detected and intrinsically preserved. Two special functions introduced in the variational approximation of the original functional permit the detection of edges and creases. The BZ model is applied first in 2D and then in 1D to accomplish different goals. In 2D, the two discontinuity functions are exploited for the detection of building candidates and roof planes; in the edge and edge\&crease maps roof edges and ridges are evident. In $1 \mathrm{D}$, the $\mathrm{BZ}$ model is used to smooth the boundary of connected regions without compromising its corners. In the procedure, other specific solutions are adopted to solve different problems, namely the detection of right-angle polygonal shapes and the modeling and refinement of roof planes.

The main feature of the procedure can be summarized as follows; a) the roof planes are labeled on a hierarchical basis, i.e. each roof plane is labeled as a part of a specific building; b) the roof planes modeling is based on the total least squares principle; $c$ ) the refinement of roof patches is based on a specific measure involving only the coordinates of candidate refinement points and the standard deviation of the TLS model fitting the patches. This choice is of particular relevance since the points considered in the refinement are edge or crease points which have very poor local properties.

The procedure is essentially global and the 2D BZ model can be applied to a whole DSM. When dealing with large files tiling can be very convenient or even necessary. In (Zanella et al., 2018) a parallel method is proposed which converges to a stationary point of the objective energy.

A limit to the procedure derives from the fact that the values of the weight parameters that appear in the object functional need to be empirically chosen. However, for a given set of parameter values, different data can be processed to detect edges and creases of the same amplitude and to smooth the data of the same amount.

The application of the procedure to a DSM interpolated from the ALS point cloud with a grid width of $25 \mathrm{~cm}$, using only the points corresponding to the last pulse, produced good results which fulfilled the objectives set out for this work. The results demonstrate the usefulness of the BZ model, the rationale of the procedure and the validity of its numerical implementation. 
In this work gridded data have been considered just because of the adoption of the finite difference method for the numerical approximation of the analytical models involved in the procedure. A different approximation method would permit the processing of sparse points; the procedure itself would remain the very same.

The propose procedure could be further developed considering height data at higher spatial resolution. As in (Grigillo and Kanjir, 2012), the application of some boundary vectorialization technique is expected to improve the performance of an extended procedure, in particular with respect to the values of the completeness and correctness indices on a per-pixel level and the geometry accuracy of object boundaries. Geometry of building footprints and roof planes could be further improved by defining special treatment of false negative pixels appearing nearby the building walls and by applying reconstruction-type algorithms.

Other extensions of the presented work can be identified. By the analysis of the areas of the edge map where $\mathbf{s}<0.8$ it could be possible to derive useful information for the detection and modeling of trees or other objects. In (Carriero et al., 2012) an extension of the BZ model is presented to tackle the problem of image inpainting. The extended model could be applied to DSM data to test its capabilities of reducing the mask effect of trees on roof planes. By changing the numerical approximation technique, from finite differences to finite elements, it could be possible to process non-gridded height data, see (Zanetti and Bruzzone, 2014). Moreover the BZ model could also be exploited for the simplification of TIN models with preserved and identified break-lines; as a starting reference see (Bourdin and Chambolle, 2000).

\section{Acknowledgement}

The Vaihingen data set was provided by the German Society for Photogrammetry, Remote Sensing and Geoinformation (DGPF), (Cramer, 2010).

The reviewers are acknowledged for their careful reading of the manuscript and for their constructive comments that leaded to improve the manuscript.

\section{References}

Ambrosio, L., Faina, L., March, R., 2001. Variational Approximation of a second order free discontinuity problem in computer vision. SIAM J. Math. Anal. 32, 1171-1197. 
Ambrosio, L., Fusco, N., Pallara, D., 2000. Functions of bounded variation and free discontinuity problems. Oxford Mathematical Monographs, Oxford University Press.

Ambrosio, L., Tortorelli, V.M., 1992. On tha approximation of free discontinuity problems. Boll. Un. Mat. Ital. B (7), 105-123.

Awrangjeb, M., Zhang, C., Fraser, C.S., 2013. Automatic extraction of building roofs using LiDAR data and multispectral imagery. ISPRS Journal of Photogrammetry and Remote Sensing 83, 1-18. doi:10.1016/j.isprsjprs.2013.05.006.

Barazzetti, L., Brovelli, M.A., Valentini, L., 2010. LiDAR digital building models for true orthophoto generation. Applied Geomatics 2, 187-196. doi:10.1007/s12518-010-0034-9.

Bellettini, G., Coscia, A., 1994a. Approximation of a functional depending on jumps and corners. Bollettino della Unione Matematica Italiana-B , $151-182$.

Bellettini, G., Coscia, A., 1994b. Discrete approximation of a free discontinuity problem. Numerical Functional Analysis Optimization 15, 201-224.

Benciolini, G., Vitti, A., 2015. Polygonal shapes detection in 3D models of complex architectures. International Archives of the Photogrammetry, Remote Sensing and Spatial Information Sciences 40, 387-392. doi:10.5194/isprsarchives-XL-5-W4-387-2015.

Biosca, J.M., Lerma, J.L., 2008. Unsupervised robust planar segmentation of terrestrial laser scanner point clouds based on fuzzy clustering methods. ISPRS Journal of Photogrammetry and Remote Sensing 63, 84-98. doi:10.1016/j.isprsjprs.2007.07.010.

Blake, A., Zisserman, A., 1987. Visual Reconstruction. MIT Press.

Borghi, A., Cannizzaro, L., Vitti, A., 2012. Advanced techniques for discontinuity detection in GNSS coordinate time-series. an Italian case study, pp. 627-634. doi:10.1007/978-3-642-20338-1_77.

Borrmann, D., Elseberg, J., Lingemann, K., Nüchter, A., 2011. The 3d hough transform for plane detection in point clouds: A review and a new accumulator design. 3D research 2, 1-13. doi:10.1007/3DRes.02(2011)3. 
Bourdin, B., Chambolle, A., 2000. Implementation of an adaptive finiteelement approximation of the mumford-shah functional. Numerische Mathematik 85, 609-646. doi:10.1007/PL00005394.

Braides, A., 2002. Gamma-convergence for Beginners. Oxford University Press.

Bredies, K., Kunisch, K., Pock, T., 2010. Total Generalized Variation. SIAM Journal of Imaging Science 3, 492-526. doi:10.1137/090769521.

Brenner, C., 2005. Building reconstruction from images and laser scanning. International Journal of Applied Earth Observation and Geoinformation 6, 187-198. doi:10.1016/j.jag.2004.10.006.

Carriero, M., Leaci, A., Tomarelli, F., 2012. Free gradient discontinuity and image inpainting. Journal of Mathematical Sciences 181, 805-819. doi:10.1007/s10958-012-0716-4.

Carriero, M., Leaci, A., Tomarelli, V., 1997. Strong minimizers of Blake \& Zisserman functional. Ann. Scuola Norm. Sup. Pisa Cl. Sci. 25, 257-285.

Chan, T., Esedoglu, S., Park, F., Yip, A., 2006. Handbook of Mathematical Models in Computer Vision. Springer, Boston. chapter Total Variation Image Restoration: Overview and Recent Developments. pp. 17-31. doi:10.1007/0-387-28831-7_2.

Chen, D., Zhang, L., Li, J., Liu, R., 2012. Urban building roof segmentation from airborne LiDAR point clouds. International Journal of Remote Sensing 33, 6497-6515. doi:10.1080/01431161.2012.690083.

Cohen-Steiner, D., Alliez, P., Desbrun, M., 2004. Variational shape approximation. ACM Transaction On Graphics 23, 905-914. doi:10.1145/1015706.1015817.

Cramer, M., 2010. The DGPF-Test on Digital Airborne Camera Evaluation Overview and Test Design. PFG Photogrammetrie, Fernerkundung, Geoinformation 2010, 73-82. doi:http://dx.doi.org/10.1127/1432$8364 / 2010 / 0041$.

De Giorgi, E., 1991. Frontiers in Pure and Applied Mathemathics, a collection of papers dedicated to J.L. Lions on the occasion of his 60th birthday. 
North-Holland P.C.. chapter Free discontinuity problems in calculus of variations. pp. 55-62.

De Giorgi, E., Carriero, M., Leaci, A., 1989. Existence theorem for a minimum problem with free discontinuity set. Archive for Rational Mechanics and Analysis 108, 195-218. doi:10.1007/BF01052971.

De Giorgi, E., Franzoni, T., 1975. Su un tipo di convergenza variazionale. Atti Accad. Naz. Lincei Rend. Cl. Sci. Mat. 8, 842-850.

Delong, A., Osokin, A., Isack, H.N., Boykov, Y., 2012. Fast approximate energy minimization with label costs. International Journal of Computer Vision 96, 1-27. doi:10.1007/s11263-011-0437-z.

Demir, N., Baltsavias, E., 2012. Automated modeling of 3D building roofs using image and LiDAR data. ISPRS Annals of Photogrammetry, Remote Sensing and Spatial Information Sciences I, 35-40. doi:10.5194/isprsannals-I-4-35-2012.

Filin, S., Pfeifer, N., 2006. Segmentation of airborne laser scanning data using a slope adaptive neighborhood. ISPRS Journal of Photogrammetry and Remote Sensing 60, 71-80. doi:10.1016/j.isprsjprs.2005.10.005.

Fischler, M.A., Bolles, R.C., 1981. Random sample consensus: A paradigm for model fitting with applications to image analysis and automated cartography. Commun. ACM 24, 381-395. doi:10.1145/358669.358692.

Goebbels, S., Pohle-Fröhlich, R., 2016. Roof Reconstruction from Airborne Laser Scanning Data Based on Image Processing Methods. ISPRS Annals of Photogrammetry, Remote Sensing and Spatial Information Sciences, 407-414doi:10.5194/isprs-annals-III-3-407-2016.

Grigillo, D., Kanjir, U., 2012. Urban Object Extraction From Digital Surface Model And Digital Aerial Images. ISPRS Annals of Photogrammetry, Remote Sensing and Spatial Information Sciences I-3, 215-220. doi:10.5194/isprsannals-I-3-215-2012.

Haala, N., Kada, M., 2010. An update on automatic 3D building reconstruction. ISPRS Journal of Photogrammetry and Remote Sensing 65, 570-580. doi:10.1016/j.isprsjprs.2010.09.006. 
Habib, A., Kim, C., 2006. Lidar-aided true orthophoto and dbm generation system, in: Innovations in 3D Geo Information Systems. Springer. Lecture Notes in Geoinformation and Cartography, pp. 47-65. doi:10.1007/978-3540-36998-1_4.

Harris, C., Stephens, M., 1988. A combined corner and edge detector., in: Alvey vision conference, Citeseer. p. 50.

Hossam, I., Boykov, Y., 2012. Energy-based geometric multi-model fitting. International Journal of Computer Vision 97, 123-147. doi:10.1007/s11263-011-0474-7.

Hough, P.V.C., 1962. Method and means for recognizing complex patterns. Patent. US 3,069,654.

Huang, H., Brenner, C., Sester, M., 2013. A generative statistical approach to automatic 3D building roof reconstruction from laser scanning data. ISPRS Journal of Photogrammetry and Remote Sensing 79, 29-43. doi:10.1016/j.isprsjprs.2013.02.004.

Jochem, A., Höfle, B., Rutzinger, M., Pfeifer, N., 2009. Automatic roof plane detection and analysis in airborne LiDAR point clouds for solar potential assessment. Sensors 9, 5241-5262. doi:10.3390/s90705241.

Jochem, A., Höfle, B., Wichmann, V., Rutzinger, M., Zipf, A., 2012. Area-wide roof plane segmentation in airborne LiDAR point clouds. Computers, Environment and Urban Systems 36, 54-64. doi:10.1016/j.compenvurbsys.2011.05.001.

Kim, K., Shan, J., 2011. Building roof modeling from airborne laser scanning data based on level set approach. ISPRS Journal of Photogrammetry and Remote Sensing 66, 484-497. doi:10.1016/j.isprsjprs.2011.02.007.

Lavoué, G., Dupont, F., 2009. Semi-sharp subdivision surface fitting based on feature lines approximation. Computers \& Graphics 33, 151-161. doi:10.1016/j.cag.2009.01.004.

Lin, C.H., Chen, J.Y., Su, P.L., Chen, C.H., 2014. Eigen-feature analysis of weighted covariance matrices for LiDAR point cloud classification. ISPRS Journal of Photogrammetry and Remote Sensing 94, 70-79. doi:http://dx.doi.org/10.1016/j.isprsjprs.2014.04.016. 
Lindeberg, T., 1998. Feature detection with automatic scale selection. International journal of computer vision 30, 79-116.

Modica, L., Mortola, S., 1977. Un esempio di Gamma-convergenza. Bollettino dell'Unione Matatematica Italiana B-14, 285-299.

Mumford, D., Shah, J., 1989. Optimal Approximations by Piecewise Smooth Functions and Associated Variational Problems. Comm. Pure Appl. Math. $42,577-685$.

Ohtake, Y., Belyaev, A., Seidel, H.P., 2004. Ridge-valley lines on meshes via implicit surface fitting. ACM Transaction on Graphics 23, 609-612. doi:10.1145/1015706.1015768.

Perona, P., Malik, J., 1990. Scale-Space and Edge Detection Using Anisotropic Diffusion. IEEE Trans. Pattern Anal. Mach. Intell. 12, 629639. doi:10.1109/34.56205.

Rottensteiner, F., 2003. Automatic generation of high-quality building models from LiDAR data. IEEE Computer Graphics and Applications 23, 42-50. doi:10.1109/MCG.2003.1242381.

Rottensteiner, F., 2010. Roof plane segmentation by combining multiple images and point clouds. International Archives of Photogrammetry, Remote Sensing and Spatial Information Sciences 38, 245-250.

Rottensteiner, F., Sohn, G., Gerke, M., Wegner, J.D., Breitkopf, U., Jung, J., 2014. Results of the ISPRS benchmark on urban object detection and 3d building reconstruction. ISPRS Journal of Photogrammetry and Remote Sensing 93, 256-271. doi:10.1016/j.isprsjprs.2013.10.004.

Rottensteiner, F., Sohn, G., Jung, J., Gerke, M., Baillard, C., Benitez, S., Breitkopf, U., 2012. The ISPRS Benchmark On Urban Object Classification And 3d Building Reconstruction. ISPRS Annals of Photogrammetry, Remote Sensing and Spatial Information Sciences I-3, 293-298. doi:10.5194/isprsannals-I-3-293-2012.

Rudin, L.I., Osher, S., Fatemi, E., 1992. Nonlinear total variation based noise removal algorithms. Physica D: Nonlinear Phenomena 60, 259-268. doi:10.1016/0167-2789(92)90242-F. 
Rutzinger, M., Rottensteiner, F., Pfeifer, N., 2009. A Comparison of Evaluation Techniques for Building Extraction From Airborne Laser Scanning. IEEE Journal of Selected Topics in Applied Earth Observations and Remote Sensing 2, 11-20. doi:10.1109/JSTARS.2009.2012488.

Sampath, A., Shan, J., 2010. Segmentation and reconstruction of polyhedral building roofs from aerial LiDAR point clouds. IEEE Transactions on Geoscience and Remote Sensing 48, 1554-1567. doi:10.1109/TGRS.2009.2030180.

Satari, M., Samadzadegan, F., Azizi, A., Maas, H.., 2012. A MultiResolution Hybrid Approach for Building Model Reconstruction from LiDAR Data. The Photogrammetric Record 27, 330-359. doi:10.1111/j.14779730.2012.00688.x.

Tarsha-Kurdi, F., Landes, T., Grussenmeyer, P., 2007a. Hough-transform and extended ransac algorithms for automatic detection of 3d building roof planes from lidar data. International Archives of Photogrammetry, Remote Sensing and Spatial Information Sciences 3, 407-412.

Tarsha-Kurdi, F., Landes, T., Grussenmeyer, P., Koeh, M., 2007b. Modeldriven and data-driven approaches using LIDAR data: analysis and comparison, in: ISPRS Workshop, Photogrammetric Image Analysis (PIA07), ISPRS. pp. 87-92.

Vitti, A., 2012a. Sigseg: a tool for the detection of position and velocity discontinuities in geodetic time-series. GPS Solutions 16, 405-410.

Vitti, A., 2012b. The Mumford-Shah variational model for image segmentation: an overview of the theory, implementation and use. ISPRS Journal of Photogrammetry and Remote Sensing 69, 50-64. doi:10.1016/j.isprsjprs.2012.02.005.

Wang, Y., Hao, W., Ning, X., Zhao, M., Zhang, J., Shi, Z., Zhang, X., 2013. Automatic segmentation of urban point clouds based on the gaussian map. The Photogrammetric Record 28, 342-361. doi:10.1111/phor.12041.

Yan, J., Shan, J., Jiang, W., 2014. A global optimization approach to roof segmentation from airborne lidar point clouds. ISPRS Journal of Photogrammetry and Remote Sensing 94, 183-193. doi:10.1016/j.isprsjprs.2014.04.022. 
Yazid, H., Arof, H., 2013. Gradient based adaptive thresholding. Journal of Visual Communication and Image Representation 24, 926-936.

Zanella, R., Porta, F., Ruggiero, V., Zanetti, M., 2018. Serial and parallel approaches for image segmentation by numerical minimization of a secondorder functional. Applied Mathematics and Computation 318, 153-175. doi:10.1016/j.amc.2017.07.021.

Zanetti, M., Bruzzone, L., 2014. Edge-crease detection and surface reconstruction from point clouds using a second-order variational model, pp. 924409-924409-11. doi:10.1117/12.2069666.

Zanetti, M., Bruzzone, L., 2017. Piecewise linear approximation of vector-valued images and curves via second-order variational model. IEEE Transactions on Image Processing 26, 4414-4429. doi:10.1109/TIP.2017.2716827.

Zanetti, M., Ruggiero, V., Miranda Jr, M., 2016. Numerical minimization of a second-order functional for image segmentation. Communications in Nonlinear Science and Numerical Simulation 36, 528-548. doi:http://dx.doi.org/10.1016/j.cnsns.2015.12.018.

Zanetti, M., Vitti, A., 2013. The Blake-Zisserman model for digital surface models segmentation, in: ISPRS Ann. Photogramm. Remote Sens. Spatial Inf. Sci., pp. 355-360. doi:10.5194/isprsannals-II-5-W2-355-2013. 